\title{
ANALYSIS OF FISH ASSEMBLAGES IN SECTORS ALONG A SALINITY GRADIENT BASED ON SPECIES, FAMILIES AND FUNCTIONAL GROUPS
}

\author{
Ana Carolina dos Passos ${ }^{1 *}$, Riguel Feltrin Contente ${ }^{2}$, Felippe Veneziani Abbatepaulo ${ }^{3}$, \\ Henry Louis Spach, Ciro Colodetti Vilar ${ }^{3}$, Jean Christophe Joyeux ${ }^{1}$, \\ Beatriz Fernanda Chinhilla Cartagena ${ }^{4}$ and Luis Fernando Fávaro ${ }^{3}$
}

${ }^{1}$ Universidade Federal do Espírito Santo

(Av. Fernando Ferrari, 514, Departamento de Oceanografia e Ecologia, 29075-910, Vitória, ES, Brasil)

${ }^{2}$ Instituto Oceanográfico da Universidade de São Paulo (Praça do Oceanográfico, 191 05508-120 São Paulo, SP, Brasil)

${ }^{3}$ Universidade Federal do Paraná

(Caixa Postal 61, 83255-976 Pontal do Paraná, PR, Brasil)

${ }^{4}$ Universidade Federal do Pará

(Al. Leandro Ribeiro, s/n, Instituto de Estudos Costeiros, 68600-000, Bragança, PA, Brasil)

*Corresponding author: anapassosoc@gmail.com

\begin{abstract}
A B S T R A C T
Here we test the effects of the east-west salinity gradient in the subtropical Paranaguá Bay Estuarine Complex (PEC) on the structure of shallow water fish fauna, determined according to taxonomic (families and species) and functional composition metrics. A total of 152 species were observed. The families with the largest number of species were the Sciaenidae, Carangidae, Haemulidae and Gobiidae. The most abundant species were Atherinella brasiliensis, Harengula clupeola, Anchoa januaria and Anchoa tricolor. Marine stragglers dominated in number of species, followed by marine migrants and estuarine species. Most species were zoobenthivores, followed by piscivores and zooplanktivores. Families and species more frequently associated with estuarine conditions dominated in the mesohaline sector, and those more frequently associated with marine conditions dominated in the euhaline sector. The fish assemblages along the estuarine salinity gradient were found to be better characterized by taxonomic metrics than by functional ones. This is most likely because individuals of all functional groups inhabit all salinity sectors, and thus these metrics are not useful for differentiating assemblages along salinity gradients. Our results differ from those of other studies in tropical and subtropical estuaries, which have emphasized the importance of functional groups in determining fish assemblages along salinity gradients.
\end{abstract}

\section{RESUMO}

Neste trabalho foi testado o efeito do gradiente de salinidade do eixo-leste oeste do sistema subtropical Complexo Estuarino da Baía de Paranaguá na estrutura dos peixes de águas rasas, determinado de acordo com as métricas taxonômica (famílias e espécies) e de composição funcional. Um total de 152 espécies foi registrado. As famílias com maior número de espécies foram Sciaenidae, Carangidae, Haemulidae e Gobiidae. As espécies mais abundantes foram A. brasiliensis, H. clupeola, A. januaria e A. tricolor. Os visitantes marinhos dominaram em número de espécies, seguidos pelos migrantes marinhos e estuarinos. A maioria das espécies são zoobentívoras, seguidas pelas piscívoras e zooplanctívoras. As famílias e espécies mais relacionadas com condições estuarinas dominaram no setor mesohalino e aquelas mais relacionadas com condições marinhas dominaram no setor euhalino. A métrica taxonômica foi mais eficiente na caracterização das assembleias de peixes ao longo do gradiente estuarino de salinidade do que a funcional. Isso ocorreu principalmente porque indivíduos de todos os grupos funcionais estiveram presentes ao longo de todos os setores de salinidade, invalidando o emprego dessa métrica na diferenciação das assembleias nos diversos setores. Nosso resultado foi diferente do encontrado em outros estuários tropicais e subtropicais, que enfatizaram a importância dos grupos funcionais na estruturação das assembleias de peixes ao longo de um gradiente de salinidade.

Descriptors: Salinity, Ichthyofauna, Estuarine use, Trophic position, Paranaguá Estuarine Complex. Descritores: Salinidade, Ictiofauna, Uso do estuário, Posição trófica, Complexo Estuarino de Paranaguá. 


\section{INTRODUCTION}

Designation of functional groups (FG) is an operational approach used to simplify the structure and dynamics of ecosystems, thus facilitating understanding of complex factors (BLONDEL, 2003). The determination of FG has been widely used to describe the structure of estuarine fish assemblages by grouping fish species according to their trophic level, reproduction strategy and use of the estuary (GARRISON; LINK, 2000; ANGEL; OJEDA, 2001; LOBRY et al., 2003; CHAVES; BOUCHEREAU, 2004; ELLIOTT et al., 2007; FRANCO et al., 2008; SELLESLAGH et al., 2009). Diversity in life cycle, habitat use and trophic position among fishes is expected to affect the functioning of the ecosystem; thus examining these factors is useful for understanding how an ecosystem functions. (ELLIOTT et al., 2007). Using a traditional taxonomic approach, along with an analysis of FG, represents a holistic approach to describing the structure of fish fauna (FRANCO et al., 2008). Moreover, because functional aspects provide important cues regarding ecosystem health, this approach may help in decisionmaking processes related to the management of estuarine areas (MATHIESON et al., 2000).

Tropical and subtropical estuaries are more complex than temperate ones, primarily due to their great biodiversity, coupled with biological productivity, high habitat diversity and multiple complex interactions among biotic and highly variable abiotic factors (SHEAVES, 2006). Despite these important biodiversity attributes, tropical estuaries are under increased human pressure because most tropical estuaries are in developing countries that have experienced accelerated coastal development in recent decades. Thus, an understanding of the functional traits of fish assemblages is critical for fully understanding ecosystem function (BLABER, 2008).

The Paranaguá Estuarine Complex (PEC), situated on the coast of Paraná state (southern Brazil), constitutes the southern sector of the large subtropical IguapeCananéia-Paranaguá estuarine system, which is part of the southern sector of Brazil's Atlantic Forest Biosphere Reserve, a global biodiversity hotspot (Fig. 1). PEC harbors a rich fish fauna of 213 species that represent a mixture between tropical Brazilian coastal fauna and temperate Argentinian and Uruguayan species (PASSOS et al., 2012). This richness is higher than, or comparable to, those of other systems located in species-rich, tropical biogeographical zones around the world, which emphasizes the importance of PEC for global biodiversity conservation (PASSOS et al., 2012). Despite this conservation status, PEC has suffered from the impact of three ports (Paranaguá, Antonina and Ponta do Félix), dredging, discharge of domestic and industrial effluents, introduction of exotic species (VITULE et al. 2006; CAIRES et al., 2007; CONTENTE et al., 2011b), and growing urbanization (LANA et al., 2001).

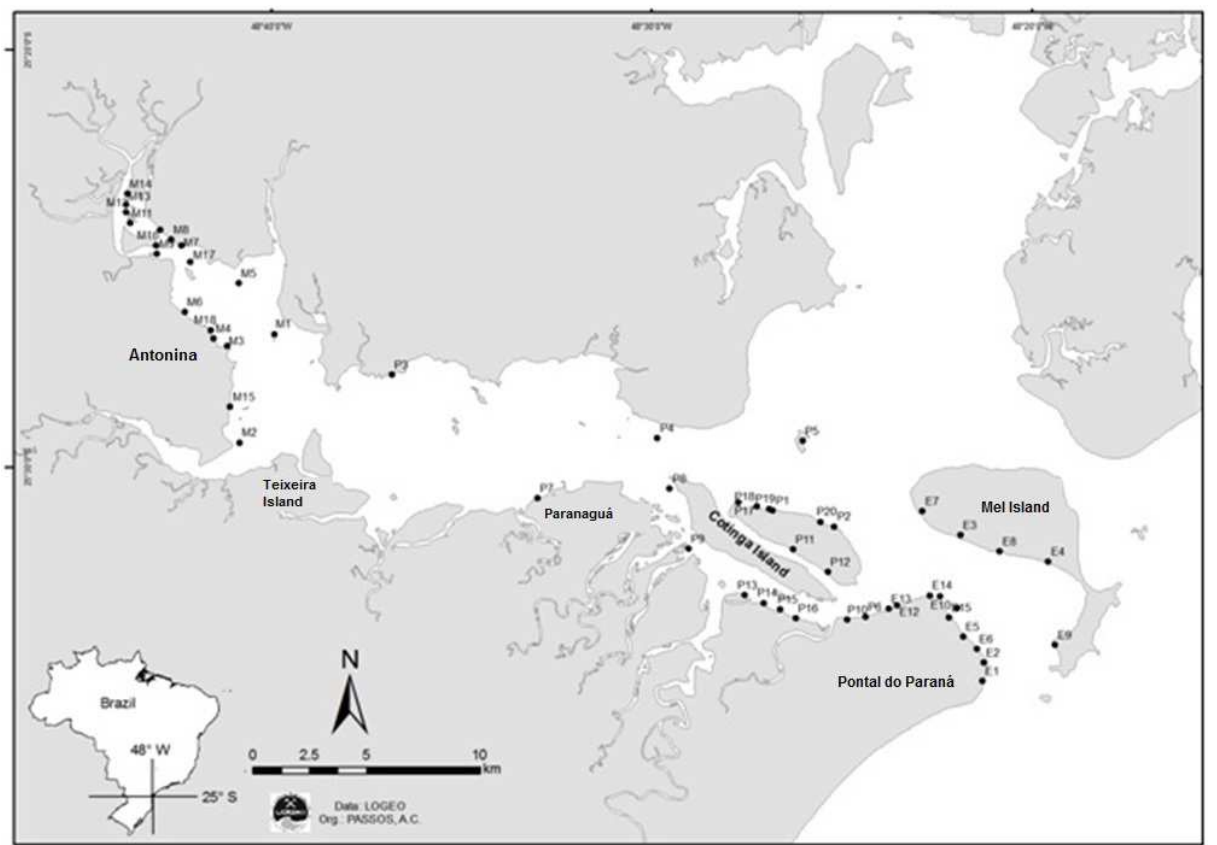

Fig. 1. Location of the sampling sites in the euhaline (E1-E15), polyhaline (P1-P20) and mesohaline (M1-M18) sectors of the east-west axis of the Paranaguá Estuarine Complex. 
The structure and composition of the fish assemblages along the east-west salinity gradient of the PEC, and their relationship to the abiotic factors of beaches (GODEFROID et al., 1997; FÉLIX et al., 2006; FÉLIX et al., 2007a, b; HACKRADT et al., 2011), rivers (CONTENTE et al., 2011a), tidal creeks (SPACH et al., 2003; SPACH et al., 2004a, b; IGNÁCIO; SPACH, 2009) and tidal flats (FALCÃO et al., 2006; FALCÃO et al., 2008; GODEFROID et al., 2003) has been widely studied, primarily using taxonomic metrics. Despite the varied studies, there is no integrated approach taking into account the spatial distribution across this gradient using taxonomic (families and species) and functional terms. Therefore, we here tested the effect of the east-west salinity gradient of the PEC on the structure of shallow water fish fauna by determining the fish taxonomic (families and species) and functional composition.

\section{Material and Methods}

\section{Study Area}

The PEC is connected to the ocean by two channels, has an east-west axis with a maximum length of $56 \mathrm{~km}$ and a maximum width of $7 \mathrm{~km}$ and a north-south axis with a maximum length of $40 \mathrm{~km}$ and a maximum width of $13 \mathrm{~km}$. The system possesses a moderate vertical salinity gradient, with semidiurnal tides with diurnal inequality, of which the maximum variation is $2.7 \mathrm{~m}$, and a consistent seasonality in circulation and stratification (KNOPPERS et al., 1987; MARONE et al., 2005). The climate of the region is transitional tropical (mean annual rainfall $=2500$ $\mathrm{mm}$ )( LANA et al., 2001), with distinctive seasonality (rainy summers and dry winters) (MARONE et al., 2005).

The east-west axis of the PEC can be divided into three salinity sectors: mesohaline (salinity between 5 and 15; Antonina region); polyhaline (salinity between 15 and 25; Teixeira Island to the eastern tip of Cotinga Island); and euhaline (salinity greater than 25; eastern part of Cotinga Island to Mel Island) (NETTO; LANA, 1996). The system is bordered by mangroves (Rhizophora mangle, Avicennia schaueriana, Laguncularia racemosa and Conocarpus erectus) (LANA et al., 2001) and salt marsh beds (Spartina alterniflora) (NETTO; LANA, 1997). For each sector, salinity, sampling sites, sampling periods and gear are provided in Table 1.

\section{Data Collection}

Unpublished data from the Fish Ecology Laboratory of the Center of Marine Studies of the Federal University of Paraná and data from published studies conducted in tidal flats on the east-west axis of the PEC were compiled. Details of the surveys can be found in the following papers: Godefroid et al. (1997), Spach et al. (2003), Godefroid et al. (2003), Spach et al. (2004a, b), Falcão et al. (2006), Félix et al. (2006), Félix et al. (2007a, b), Falcão et al. (2008), Ignácio and Spach (2009), Contente et al. (2011a), and Hackradt et al. (2011). A total of 52 sites (18 in the mesohaline, 31 in the polyhaline and 22 in the euhaline zone) were considered (Fig. 1).

Fish species nomenclature was confirmed by comparison with information from Craig and Hastings (2007), Sheaves and Craig (2007), Eschmeyer (2010), Carvalho-Filho et al. (2010) and Figueiredo et al. (2010). Mugil sp. was used for the species usually identified under the invalid name Mugil gaimardianus (MENEZES et al., 2003). Species were allocated to the following estuarine use of FG according to Elliott et al. (2007): marine stragglers (MS; species that spawn at sea and enter estuaries in low numbers, are considered stenohaline and are found at salinities of approximately 35), marine migrants (MM; species that spawn at sea and enter estuaries in large numbers as juveniles; considered euryhaline), estuarine species (ES), anadromous (AN; species that undergo their growth at sea and migrate into rivers to spawn), catradomous (CA; species that live in freshwater and migrate to marine environments to spawn), amphidromous (AM; migrate between the sea and freshwater with neither migration related to reproduction) and freshwater migrants (FM; found in moderate numbers in estuaries; considered oligohalines). Species were allocated to the following feeding mode functional groups (ELLIOTT et al. 2007): zooplanktivore (ZP; feeding on zooplankton), detritivore (DV; feeding on detritus and/or microphytobenthos), herbivore (HV; grazing on macroalgae, macrophytes or phytoplankton), omnivore (OV; feeding on filamentous algae, macrophytes, peryphyton, epifauna and infauna), piscivore (PV; feeding on finfish and large nektonic invertebrates), zoobenthivore ( $\mathrm{ZB}$; feeding on invertebrates that live just above, on or in the sediment) and opportunist (OP; feeding on a diverse range of food).

\section{Data Analysis}

Because our descriptive distribution models were spatial, individuals from all the surveys for each species were grouped first by sampling point; then the percentage of species within a family, the frequency of occurrence per species, the percentage of individuals per species, and the percentage of species and individuals per functional group were calculated for each sampling point. We expressed the number of individuals as a percentage in order to standardize the scale of the abundance estimates, which were inherently biased due to the different sampling protocols. 
Table 1. Sampling sites in each salinity sector, with the respective salinity ranges, periods and sampling methods, as well as the number of families, species and individuals. Codes for sampling nets are as follows: $(1)=$ seine $(15 \mathrm{~m} \times 2.6 \mathrm{~m}, 2 \mathrm{~m}$ bag and 0.5 mesh), (2) = seine $(15 \mathrm{~m} \times 2.6 \mathrm{~m}, 2 \mathrm{~m}$ bag and $1 \mathrm{~cm} \mathrm{mesh}),(3)=$ seine $(30 \mathrm{~m} \mathrm{x} 2 \mathrm{~m}, 2 \mathrm{~m}$ bag and $0.5 \mathrm{~cm}$ mesh), (4) = seine (15 $\mathrm{m} \times 2 \mathrm{~m}$ and $1 \mathrm{~cm}$ mesh), (5) = capéchade with barrier $(20 \mathrm{~m} \times 2.0 \mathrm{~m}, 13 \mathrm{~mm}$ mesh and 3 minnow traps, with 13.0 to $6.0 \mathrm{~mm}$ mesh), (6) = seine ( $9 \mathrm{~m} \times 2.5 \mathrm{~m}$, wings with $13 \mathrm{~mm}$ mesh and bag with $5 \mathrm{~mm}$ mesh), (7) = seine (15 m x $1.60 \mathrm{~m}$, wings with 13 $\mathrm{mm}$ mesh and bag with $5 \mathrm{~mm}$ mesh $),(8)=$ seine $(15 \mathrm{~m} \times 2 \mathrm{~m}, 2.5 \mathrm{~cm} \mathrm{mesh}),(9)=$ seine $(30 \mathrm{~m} \times 3 \mathrm{~m}$ and $0.5 \mathrm{~cm} \mathrm{mesh}),(10)=$ seine (18 $\mathrm{m} \times 2 \mathrm{~m}$ and 1 to $2 \mathrm{~mm}$ mesh), (11) = seine ( $30 \mathrm{~m} \mathrm{x} 2 \mathrm{~m}, 2 \mathrm{~m}$ bag and $0.5 \mathrm{~cm} \mathrm{mesh})$.

\begin{tabular}{|c|c|c|c|c|c|c|c|}
\hline Sector & Site & Salinity & Sampling period & Sampling method & No. of families & No. of species & No. of individuals \\
\hline Mesohaline & M1 & $9.0-27.0$ & Oct/2005 - Sep/2006 & (1) and (2) & 3 & 3 & 3 \\
\hline Mesohaline & M2 & $10.0-27.0$ & Oct/2005 - Sep/2006 & (1) and (2) & 12 & 18 & 190 \\
\hline Mesohaline & M3 & $12.0-27.0$ & Oct/2005 - Sep/2006 & (1) and (2) & 13 & 23 & 907 \\
\hline Mesohaline & M4 & $10.0-26.0$ & Oct $/ 2005-\mathrm{Sep} / 2006$ & (1) and (2) & 13 & 20 & 385 \\
\hline Mesohaline & M5 & $4.0-25.5$ & Oct $/ 2005-\mathrm{Sep} / 2006$ & (1) and (2) & 11 & 18 & 784 \\
\hline Mesohaline & M6 & $0.0-23.0$ & Oct/2005 - Sep/2006 & (1) and (2) & 15 & 18 & 1068 \\
\hline Mesohaline & M7 & $0.0-25.0$ & Oct/2005 - Sep/2006 & (1) and (2) & 9 & 10 & 168 \\
\hline Mesohaline & M8 & $0.0 .-20.5$ & Oct/2005 - Sep/2006 & (1) and (2) & 11 & 13 & 246 \\
\hline Mesohaline & M9 & $2.0-21.0$ & Oct $/ 2005-\mathrm{Sep} / 2006$ & (1) and (2) & 12 & 16 & 471 \\
\hline Mesohaline & M10 & $0.0-19.0$ & Oct/2005 - Sep/2006 & (1) and (2) & 13 & 22 & 597 \\
\hline Mesohaline & M11 & $0.0-20.0$ & Oct/2005 - Sep/2006 & (1) and (2) & 16 & 20 & 1044 \\
\hline Mesohaline & M12 & $0.0-19.0$ & Oct $/ 2005-\mathrm{Sep} / 2006$ & (1) and (2) & 13 & 22 & 662 \\
\hline Mesohaline & M13 & $0.0-18.0$ & Oct/2005 - Sep/2006 & (1) and (2) & 14 & 22 & 354 \\
\hline Mesohaline & M14 & $0.0-15.0$ & Oct/2005 - Sep/2006 & (1) and (2) & 15 & 24 & 417 \\
\hline Mesohaline & M15 & $0.0-15.0$ & Oct/2005 - Sep/2006 & (1) and (2) & 14 & 22 & 1715 \\
\hline Mesohaline & M16 & $0.0-8.0$ & $\mathrm{Apr} / 2000-\mathrm{Mar} / 2001$ & (3) & 29 & 34 & 22867 \\
\hline Mesohaline & M17 & $2.0-10.0$ & $\mathrm{Apr} / 2000-\mathrm{Mar} / 2001$ & (3) & 22 & 32 & 5830 \\
\hline Mesohaline & M18 & $12.0-24.0$ & $\mathrm{Apr} / 2000-\mathrm{Mar} / 2001$ & (3) & 21 & 32 & 3967 \\
\hline Polihaline & P1 & $25.0-34.0$ & Mar/2006 - Mar/2007 & (4) & 25 & 50 & 2155 \\
\hline Polihaline & $\mathrm{P} 2$ & $25.0-34.0$ & Mar/2006 - Mar/2007 & (4) & 23 & 48 & 3968 \\
\hline Polihaline & P3 & $17.0-29.0$ & Jun/2005 - May/2006 & (1) & 15 & 34 & 3649 \\
\hline Polihaline & P4 & $20.0-32.0$ & Jun/2005 - May/2006 & (1) & 16 & 38 & 4225 \\
\hline Polihaline & P5 & $23.0-33.0$ & Jun/2005 - May/2006 & (1) & 31 & 58 & 2949 \\
\hline Polihaline & P6 & $20.0-34.0$ & $\mathrm{Jul} / 2006$ - Jun/2007 & (5) & 31 & 66 & 39709 \\
\hline Polihaline & $\mathrm{P} 7$ & $20.0-28.0$ & Aug/2003 - Jun/2004 & (6) and (7) & 9 & 14 & 614 \\
\hline Polihaline & P8 & $16.0-27.0$ & Aug/2003 - Jun/2004 & (6) and (7) & 14 & 22 & 2431 \\
\hline Polihaline & P9 & $17.0-28.0$ & Aug/2003 - Jun/2004 & (6) and (7) & 14 & 20 & 1284 \\
\hline Polihaline & P10 & $23.0-29.0$ & Aug/2003 - Jun/2004 & (6) and (7) & 13 & 21 & 1566 \\
\hline Polihaline & P11 & $24.0-33.0$ & $\mathrm{Apr} / 2000-\mathrm{Mar} / 2001$ & (3) & 23 & 35 & 7949 \\
\hline Polihaline & $\mathrm{P} 12$ & $25.0-33.0$ & $\mathrm{Apr} / 2000-\mathrm{Mar} / 2001$ & (3) & 27 & 39 & 15328 \\
\hline Polihaline & $\mathrm{P} 13$ & $15.0-30.0$ & Jul/2005 - Dec/2006 & (7) & 11 & 15 & 648 \\
\hline Polihaline & P14 & $17.5-33.0$ & Jul/2005 - Dec/2006 & (7) & 15 & 24 & 931 \\
\hline Polihaline & P15 & $18.0-33.0$ & Jul/2005 - Dec/2006 & (7) & 15 & 24 & 504 \\
\hline Polihaline & P16 & $17.0-34.0$ & $\mathrm{Jul} / 2005-\mathrm{Dec} / 2006$ & (7) & 7 & 11 & 527 \\
\hline Polihaline & P17 & $19.8-28.3$ & Aug/2010 - Apr/2011 & (8) & 10 & 12 & 854 \\
\hline Polihaline & P18 & $19.6-27.1$ & Aug/2010 - Apr/2011 & (8) & 14 & 17 & 685 \\
\hline Polihaline & P19 & $20.8-27.2$ & Aug/2010 - Apr/2011 & (8) & 12 & 15 & 2143 \\
\hline Polihaline & $\mathrm{P} 20$ & $23.8-28.0$ & Aug/2010 - Apr/2011 & (8) & 14 & 21 & 2925 \\
\hline Euhaline & E1 & $33.0-36.0$ & Jun/2004 - May/2006 & (1) & 22 & 55 & 23645 \\
\hline Euhaline & E2 & $25.0-34.0$ & Jun/2004 - May/2005 & (1) & 23 & 47 & 8847 \\
\hline Euhaline & E3 & $25.0-35.0$ & May/2000 - Apr/2001 & (9) & 23 & 49 & 9243 \\
\hline Euhaline & E4 & $25.0-35.0$ & May/2000 - Apr/2001 & (9) & 23 & 49 & 2822 \\
\hline Euhaline & E5 & $19.0-35.0$ & Apr/2000 - May/2001 & (3) & 19 & 30 & 3069 \\
\hline Euhaline & E6 & $28.0-36.0$ & $\mathrm{Apr} / 2000$ - May/2001 & (3) & 19 & 31 & 2557 \\
\hline Euhaline & E7 & $23.0-34.0$ & Jun/2005 - May/2006 & (1) & 16 & 37 & 1985 \\
\hline Euhaline & E8 & $24.0-35.0$ & Jun/2005 - May/2006 & (1) & 17 & 31 & 1082 \\
\hline Euhaline & E9 & $25.5-33.0$ & Jun/2005 - May/2006 & (1) & 15 & 28 & 2551 \\
\hline Euhaline & E10 & $27.1-34.8$ & May/1993 - Apr/1994 & (10) & 21 & 51 & 1713 \\
\hline Euhaline & E11 & $19.0-35.0$ & May/1993 - Apr/1994 & (10) & 32 & 71 & 13106 \\
\hline Euhaline & E12 & $11.0-35.0$ & Aug/1998 - Jul/1999 & (11) & 49 & 118 & 63165 \\
\hline Euhaline & E13 & $20.6-30.1$ & Aug/2010 - Apr/2011 & (8) & 9 & 12 & 464 \\
\hline \multirow[t]{2}{*}{ Euhaline } & E14 & $22.9-31.1$ & Aug/2010 - Apr/2011 & (8) & 7 & 12 & 1126 \\
\hline & E15 & $11.6-32.1$ & Aug/2010 - Apr/2011 & (8) & 10 & 12 & 242 \\
\hline
\end{tabular}

Non-metric multidimensional scaling (nMDS) analyses were used to assess the effect of the salinity sectors on fish taxonomic composition and functional groups. The difference in taxonomic composition and FG among salinity sectors were tested by analysis of similarity (ANOSIM). In addition to the significance level, an $\mathrm{R}$ value is calculated for the ANOSIM, which indicates the magnitude of the separation between groups. $R$-values, which range from 0 to 1 , indicate that groups are clearly distinct if $\mathrm{R}>0.75$. If $\mathrm{R}>0.5$, the groups overlap but can be differentiated, and if $R<0.25$, groups are typically 
indistinguishable (CLARKE; GORLEY, 2006). Analysis of similarity of percentages (SIMPER) was applied to identify the families, species and guilds responsible for such differences (CLARKE; WARWICK, 2001).

\section{RESULTS}

Taxonomic Distribution and Functional Groups

A total of 152 species (149 Actinopterygii and 3 Elasmobranchii), distributed in 19 orders and 51 families, were recorded along the east-west axis of the PEC. Most individuals were captured in the euhaline sector (50\% - 131 species), while the polyhaline and mesohaline sectors accounted for 35\% (109 species) and $15 \%$ (70 species) of individuals, respectively. A total of 55 species were ubiquitous along the east-west PEC axis and 36, 7 and 6 were found exclusively in the euhaline, polyhaline, and mesohaline sectors, respectively (Table 2).

Most of the families had a small number of species ( 1 to 5 species), with $c .43 \%$ represented by a single species. The families with the largest number of species were Sciaenidae (19), followed by Carangidae (15), Haemulidae (8) and Gobiidae (8), which represented $33 \%$ of the species total. The most abundant species throughout the east-west axis were A. brasiliensis (16.6\%), H. clupeola (15.9\%), A. januaria (10.3\%) and A. tricolor (10.2\%). In the mesohaline sector, the most abundant species were $A$. januaria (58\%), A. brasiliensis (10.0\%), D. rhombeus (7\%) and C. schufeldti (6.6\%), while in the polyhaline sector, A. brasiliensis (30\%), H. clupeola (15.3\%) and A. lyolepis $(13.1 \%)$ were abundant, and in the euhaline sector, H. clupeola (21.1\%), A. tricolor (17.8\%), A. brasiliensis $(8.8 \%)$ and A. surinamensis $(8.1 \%)$ were the most abundant.

Marine stragglers dominated in number of species $(41 \%)$, followed by marine migrants $(25 \%)$ and estuarine species (3\%, 35 species). Each of the other functional groups (i.e., amphidromous, anadromous, catadromous, estuarine migrant, estuarine resident, and freshwater migrant) accounted for less than $2 \%$ of the species. The estuarine species dominated in number of individuals $(50 \%)$, followed by marine stragglers $(30 \%)$ and marine migrants $(15 \%)$. The zoobenthivores had the greatest percentage of species per trophic functional group (50\%), followed by piscivores (18\%) and zooplanktivores $(16 \%)$. Each of the other functional groups (i.e., detritivores, herbivores, omnivores, and opportunists) represented less than $4 \%$ of the taxa. The zooplanktivores had the most individuals per trophic group (40\%), followed by the opportunists $(28 \%)$ and zoobenthivores $(21 \%)$.
A total of 16 species were not assigned to any functional group due to a complete lack of information about their feeding habits and estuarine habitat use patterns.

\section{The Effect of Salinity Sector on Fish Fauna}

The ordination of sampling points based on presence/absence of families (Fig. 2), and corresponding ANOSIM $(R$-Global $=0.326, P<0.01)$, showed that the polyhaline and euhaline sectors almost completely overlapped $(R=0.152 ; P<0.01)$ and the polyhaline and mesohaline sectors strongly overlapped $(R=0.259 ; P<0.01)$. There was a large difference in the families present between the mesohaline and euhaline sectors $(R=0.596 ; P<0.01)$. Families with species more suited to estuarine conditions (e.g., Atherinopsidae, Engraulidae, and Gerreidae) dominated in the mesohaline sector and those with species more suited to marine conditions (e.g., Carangidae, Clupeidae, and Tetraodontidae) dominated in the euhaline sector (Table 3).

The ordination of sampling points based on the presence/absence of species (Fig. 3), and associated ANOSIM, revealed consistent separation among salinity sectors $(R$-Global $=0.502 ; P<0.01)$. The greatest differences occurred between the mesohaline and euhaline sectors $(R=0.813 ; P<0.01)$, followed by those between the mesohaline and polyhaline sectors $(R=0.548 ; P<0.01)$. The polyhaline and euhaline sectors were indistinguishable $(R=0.131 ; P<0.05)$. The species most responsible for the differentiation of the mesohaline and euhaline sectors were Trachinotus falcatus, Harengula clupeola and Trachinotus carolinus (Table 4). Those most responsible for the differentiation of the mesohaline and polyhaline sectors were Anchoa tricolor, Sphoeroides greeleyi and Ctenogobius shufeldti.

The ordination of sampling points based on the percentage of individuals per species (Fig. 4), and associated ANOSIM, revealed that each salinity sector differed significantly, although with some overlap $(R$ Global $=0.437 ; P<0.01)$. The paired comparisons indicated significant differences between the mesohaline and euhaline sectors $(R=0.622 ; P<0.01)$, overlap, but distinction, between the mesohaline and polyhaline sectors $(R=0.479 ; P<0.01)$ and no distinction between the polyhaline and euhaline sectors $(R=0.205 ; P<0.01)$. Following the same tendency observed in the family analysis, there was a progressive increase in the occurrence of species more suited to estuarine conditions (i.e., A. brasiliensis, A. januaria, and $D$. rhombeus ) in the mesohaline sector and an increase in species more suited to marine conditions (i.e., A. tricolor, H. clupeola and $T$. falcatus) in the euhaline sector (Table 5). 
Table 2. Species and their respective families in alphabetical order, as well as abundance in each of the sectors, estuarine use and trophic guilds and the references used to classify each species. Estuarine use: $\mathrm{AN}=$ anadromous, $\mathrm{AM}=$ amphidromous, $\mathrm{CA}$ = catadromous, $\mathrm{EM}=$ estuarine migrant, $\mathrm{ER}=$ estuarine resident, $\mathrm{ES}=$ estuarine, $\mathrm{FM}=$ freshwater migrant, $\mathrm{MM}=$ marine migrant, $\mathrm{MS}=$ marine straggler and $\mathrm{SC}=$ semi-catadromous. Trophic guilds: $\mathrm{ZP}=$ zooplanktivorous, $\mathrm{DV}=$ detritivorous, $\mathrm{HV}=$ herbivorous, $\mathrm{OV}=$ omnivorous, $\mathrm{PV}=$ piscivorous, $\mathrm{ZB}=$ zoobenthic, $\mathrm{OP}=$ opportunistic. Estuarine use references: $a-$ Reis Filho et al. (2010); $b$ - Barletta \& Blaber (2007); c - Barletta et al. (2008); $d$ - Froese \& Pauly (2010); e - Vilar et al. (2011); $f$ Garcia \& Vieira (2001). Trophic guild references: 1 - Froese \& Pauly (2010); 2 - Alves \& Filho (1996); 3 - Barletta \& Blaber (2007); 4 - Guedes \& Araujo (2008); 5 - Araujo (1984); 6 - Contente et al. (2011); 7 - Piedras \& Pouey (2005); 8 - Cassemiro et al. (2003); 9 - Randall (1967); 10 - Stefanoni (2008); 11- Chaves \& Bouchereau (2004); 12 - Zahorcsak et al. (2000); 13 Contente et al. (2009); 14 - Teixeira (1997); 15 - Figueiredo \& Menezes (1978); 16 - Sacardo \& Rossi-Wongtschowski (1991); 17 - Guedes \& Araujo (2008); 18 - Sergipense et al. (1999); 19 - Bortoluzzi et al. (2006); 20 - Hayse (1990); 21 - Hiatt \& Strasburg (1960); 22 - Chaves \& Otto (1998); 23 - Contente (2008); 24 - Corrêa \& Uieda (2007); 25 - Gegg \& Fleeger (1997); 26 - Vieira (1991); 27 - Nagelkerken et al. (2001); 28 - Castillo-Rivera et al. (2000); 29 - Elliot et al. (2007); 30 - Figueiredo (1977); 31 - Vendel \& Chaves (1998); 32 - Chaves \& Umbria (2003); 33 - Soares \& Vazzoler (2001); 34 - Chaves \& Vendel (1998); 35 - Teixeira \& Haimovici (1989); 36 - Cervigón (1994).

\begin{tabular}{|c|c|c|c|c|c|c|}
\hline \multirow[t]{2}{*}{ Families } & \multirow[t]{2}{*}{ Species } & \multirow[t]{2}{*}{$\begin{array}{l}\text { Mesohaline } \\
\text { sector }\end{array}$} & \multirow[t]{2}{*}{$\begin{array}{l}\text { Polihaline } \\
\text { Sector }\end{array}$} & \multirow[t]{2}{*}{$\begin{array}{l}\text { Euhaline } \\
\text { Sector }\end{array}$} & \multicolumn{2}{|l|}{ Guilds } \\
\hline & & & & & $\begin{array}{c}\text { Estuarine } \\
\text { use }\end{array}$ & Tropic \\
\hline \multirow[t]{4}{*}{ Achiridae } & Achirus declivis & 1 & 6 & 0 & $\mathrm{ES}^{\mathrm{a}}$ & $\mathrm{ZB}^{1}$ \\
\hline & Achirus lineatus & 41 & 92 & 4 & $E S^{\mathrm{a}, \mathrm{b}, \mathrm{c}}$ & $\mathrm{ZB}^{2,3,4}$ \\
\hline & Trinectes microphthalmus & 0 & 0 & 1 & $\mathrm{MM}^{\mathrm{a}}$ & \\
\hline & Trinectes paulistanus & 19 & 2 & 1 & $\mathrm{ES}^{\mathrm{a}}$ & $\mathrm{ZB}^{1,2}$ \\
\hline Albulidae & Albula vulpes & 0 & 121 & 310 & $\mathrm{AM}^{\mathrm{a}, \mathrm{d}}$ & $\mathrm{ZB}^{1}$ \\
\hline Argentinidae & Glossanodon pygmaeus & 0 & 0 & 3 & $\mathrm{ES}^{\mathrm{d}}$ & $\mathrm{ZB}^{1}$ \\
\hline \multirow[t]{2}{*}{ Ariidae } & Cathorops spixii & 10 & 129 & 237 & $\mathrm{ES}^{\mathrm{b}, \mathrm{c}}$ & $\mathrm{ZB}^{2,3}$ \\
\hline & Genidens genidens & 478 & 51 & 994 & $\mathrm{MM}^{\mathrm{d}, \mathrm{e}}$ & $\mathrm{ZB}^{1,5}$ \\
\hline \multirow[t]{3}{*}{ Atherinopsidae } & Atherinella brasiliensis & 4182 & 28883 & 11957 & $\mathrm{ES}^{\mathrm{a}, \mathrm{d}, \mathrm{e}, \mathrm{f}, \mathrm{e}}$ & $\mathrm{OP}^{1,6}$ \\
\hline & Membras dissimilis & 0 & 0 & 72 & & \\
\hline & Odontesthes bonariensis & 0 & 0 & 2001 & $\mathrm{FM}^{\mathrm{e}}$ & $\mathrm{ZP}^{1,7,8}$ \\
\hline Batrachoididae & Opsanus beta & 1 & 2 & 0 & $\mathrm{ES}^{\mathrm{d}}$ & \\
\hline \multirow[t]{2}{*}{ Belonidae } & Strongylura marina & 5 & 79 & 260 & $\mathrm{MM}^{\mathrm{a}, \mathrm{e}}$ & $\mathrm{PV}^{1,9}$ \\
\hline & Strongylura timucu & 15 & 85 & 171 & $\mathrm{MM}^{\mathrm{a}, \mathrm{b}}$ & $\mathrm{PV}^{3}$ \\
\hline Blennidae & Parablennius pilicornis & 1 & 0 & 0 & MS & $\mathrm{ZB}^{1}$ \\
\hline \multirow[t]{15}{*}{ Carangidae } & Caranx hippos & 0 & 1 & 0 & $\mathrm{MS}^{\mathrm{a}, \mathrm{c}, \mathrm{e}}$ & $\mathrm{PV}^{1,2,9}$ \\
\hline & Caranx latus & 2 & 3 & 29 & $\mathrm{MM}^{\mathrm{a}, \mathrm{c}}$ & $\mathrm{ZB}^{2,3}$ \\
\hline & Caranx ruber & 0 & 0 & 53 & MS & $\mathrm{PV}^{1}$ \\
\hline & Chloroscombrus chrysurus & 2 & 111 & 209 & $\mathrm{MS}_{\mathrm{a}, \mathrm{c}, \mathrm{e}}$ & $\mathrm{OP}^{1,10}$ \\
\hline & Oligoplites palometa & 0 & 8 & 3 & $\mathrm{MM}^{\mathrm{a}, \mathrm{e}}$ & $\mathrm{PV}^{1,2}$ \\
\hline & Oligoplites saliens & 16 & 42 & 943 & MM & $\mathrm{ZP}^{1,10}$ \\
\hline & Oligoplites saurus & 135 & 80 & 2271 & $\mathrm{MM}^{\mathrm{a}, \mathrm{e}}$ & $\mathrm{PV}^{1,2,9}$ \\
\hline & Selene setapinnis & 0 & 0 & 45 & $\mathrm{MS}^{\mathrm{a}}$ & $\mathrm{PV}^{1}$ \\
\hline & Selene vomer & 3 & 45 & 116 & $\mathrm{MM}^{\mathrm{a}, \mathrm{b}, \mathrm{c}, \mathrm{e}}$ & $\mathrm{ZB}^{2,3,11}$ \\
\hline & Seriola lalandi & 0 & 0 & 8 & MS & $\mathrm{PV}^{1}$ \\
\hline & Trachinotus carolinus & 0 & 201 & 5573 & $M S^{e}$ & $\mathrm{ZB}^{1,10,12}$ \\
\hline & Trachinotus falcatus & 11 & 335 & 2418 & $\mathrm{MS}^{\mathrm{a}, \mathrm{e}}$ & $\mathrm{ZB}^{2,10,12}$ \\
\hline & Trachonotus goodei & 0 & 0 & 266 & MS & $\mathrm{ZB}^{1,12}$ \\
\hline & Trachinotus marginatus & 0 & 0 & 27 & MS & \\
\hline & Uraspis secunda & 0 & 1 & 1 & MS & \\
\hline \multirow[t]{2}{*}{ Centropomidae } & Centropomus parallelus & 461 & 50 & 20 & $\mathrm{ES}^{\mathrm{a}}$ & $\mathrm{ZB}^{2,13}$ \\
\hline & Centropomus undecimalis & 1 & 18 & 2 & $\mathrm{ES}^{\mathrm{a}, \mathrm{e}}$ & $\mathrm{PV}^{2,14}$ \\
\hline Clinidae & Ribeiroclinus eingenmanni & 0 & 0 & 9 & MS & \\
\hline \multirow[t]{7}{*}{ Clupeidae } & Brevoortia sp. & 0 & 0 & 1 & & $\mathrm{ZP}$ \\
\hline & Chirocentrodon bleekerianus & 0 & 0 & 8 & MS & $\mathrm{ZP}$ \\
\hline & Harangula clupeola & 14 & 14602 & 28562 & $\mathrm{MS}^{\mathrm{a}, \mathrm{e}}$ & $\mathrm{ZP}^{1,10,15}$ \\
\hline & Harengula jaguana & 0 & 0 & 29 & MS & $\mathrm{ZP}^{1}$ \\
\hline & Opistonema oglinum & 11 & 1097 & 3927 & $\mathrm{MS}^{\mathrm{a}, \mathrm{e}}$ & $\mathrm{ZP}^{1,2,9}$ \\
\hline & Platanichthys platana & 96 & 4 & 9 & $E^{\mathrm{d}}$ & $\mathrm{ZP}^{1,2}$ \\
\hline & Sardinella brasiliensis & 0 & 985 & 7433 & $\mathrm{MS}^{\mathrm{e}}$ & $\mathrm{ZP}^{1,16}$ \\
\hline Cynoglossidae & Symphurus tesselatus & 24 & 3 & 2 & $\mathrm{MM}^{\mathrm{c}, \mathrm{e}}$ & $\mathrm{ZB}^{1,17}$ \\
\hline Dactylopteridae & Dactylopterus volitans & 0 & 1 & 7 & $\mathrm{MS}^{\mathrm{a}, \mathrm{e}}$ & $\mathrm{ZB}^{1,9}$ \\
\hline Diodontidae & Chilomycterus spinosus & 42 & 83 & 34 & $\mathrm{ES}^{\mathrm{a}}$ & $\mathrm{ZB}^{1,2,11}$ \\
\hline Eleotridae & Guavina guavina & 1 & 2 & 0 & $\mathrm{ES}^{\mathrm{b}, \mathrm{d}}$ & $\mathrm{ZB}^{1,3}$ \\
\hline Elopidae & Elops saurus & 0 & 2 & 21 & $\mathrm{AM}^{\mathrm{d}}$ & $\mathrm{PV}^{1,2}$ \\
\hline \multirow[t]{6}{*}{ Engraulidae } & Anchoa filifera & 0 & 0 & 1 & $\mathrm{ES}^{\mathrm{d}}$ & $\mathrm{ZP}$ \\
\hline & Anchoa januaria & 24157 & 2733 & 1106 & $\mathrm{ES}^{\mathrm{a}, \mathrm{d}, \mathrm{e}}$ & $\mathrm{ZP}^{10}$ \\
\hline & Anchoa lyolepis & 13 & 12415 & 4082 & $\mathrm{ES}^{\mathrm{a}}$ & $\mathrm{ZP}^{1}$ \\
\hline & Anchoa tricolor & 507 & 3252 & 24127 & $\mathrm{ES}^{\mathrm{e}}$ & $\mathrm{ZP}^{1}$ \\
\hline & Cetengraulis edentulus & 144 & 2585 & 901 & $\mathrm{MM}^{\mathrm{a}, \mathrm{b}, \mathrm{e}}$ & $\mathrm{ZP}^{3,11,18}$ \\
\hline & Engraulis anchoita & 0 & 39 & 0 & $\mathrm{MS}^{\mathrm{d}}$ & $\mathrm{ZP}^{1}$ \\
\hline
\end{tabular}




\begin{tabular}{|c|c|c|c|c|c|c|}
\hline & Lycengraulis grossidens & 12 & 114 & 3273 & $\mathrm{AN}^{\mathrm{a}, \mathrm{d}, \mathrm{e}}$ & $\mathrm{ZP}^{2,19}$ \\
\hline Ephippidae & Chaetodipterus faber & 8 & 73 & 321 & $\mathrm{MS}^{\mathrm{a}, \mathrm{b}, \mathrm{e}}$ & $\mathrm{HV}^{2,3,20}$ \\
\hline \multirow[t]{2}{*}{ Fistularidae } & Fistularia petimba & 0 & 4 & 9 & $\mathrm{ES}^{\mathrm{e}}$ & $\mathrm{PV}^{21}$ \\
\hline & Fistularia tabacaria & 0 & 3 & 60 & $\mathrm{MM}^{\mathrm{a}, \mathrm{d}}$ & $\mathrm{ZP}$ \\
\hline \multirow[t]{7}{*}{ Gerreidae } & Diapterus auratus & 0 & 93 & 0 & $\mathrm{EM}^{\mathrm{a}, \mathrm{d}}$ & $\mathrm{ZB}^{1}$ \\
\hline & Diapterus rhombeus & 2924 & 1804 & 109 & $\mathrm{ES}^{\mathrm{e}}$ & $\mathrm{ZB}^{1,2,22}$ \\
\hline & Eucinostomus argenteus & 54 & 2461 & 6177 & $\mathrm{MM}^{\mathrm{a}, \mathrm{e}}$ & $\mathrm{ZB}^{2,23}$ \\
\hline & Eucinostomus gula & 0 & 383 & 415 & $\mathrm{MM}^{\mathrm{a}, \mathrm{d}, \mathrm{e}}$ & $\mathrm{ZB}^{1,12}$ \\
\hline & Eucinostomus melanopterus & 2322 & 3321 & 240 & $\mathrm{MM}^{\mathrm{a}, \mathrm{e}}$ & $\mathrm{ZB}^{1,23}$ \\
\hline & Eugerres brasilianus & 4 & 11 & 0 & $\mathrm{MM}^{\mathrm{a}}$ & $\mathrm{ZB}^{1,2}$ \\
\hline & Ulaema lefroyi & 0 & 102 & 693 & $E S^{d}$ & $\mathrm{ZP}^{1}$ \\
\hline Gobiesocidae & Gobiesox strumosus & 0 & 0 & 1 & $\mathrm{ES}^{\mathrm{d}}$ & \\
\hline \multirow[t]{8}{*}{ Gobiidae } & Bathygobius soporator & 83 & 186 & 17 & $\mathrm{MM}^{\mathrm{a}, \mathrm{e}}$ & $\mathrm{ZB}^{1,2,24}$ \\
\hline & Ctenogobius boleosoma & 4 & 19 & 1 & $\mathrm{AM}^{\mathrm{a}, \mathrm{d}, \mathrm{e}}$ & $\mathrm{ZB}^{1,25}$ \\
\hline & Ctenogobius shufeldti & 2764 & 42 & 0 & ER & ZB \\
\hline & Ctenogobius smaragdus & 16 & 6 & 2 & $\mathrm{ES}^{\mathrm{a}, \mathrm{b}}$ & $\mathrm{ZB}^{2,3}$ \\
\hline & Ctenogobius stigmaticus & 1 & 3 & 0 & $\mathrm{ES}^{\mathrm{a}, \mathrm{b}, \mathrm{c}, \mathrm{e}}$ & $\mathrm{ZB}^{3}$ \\
\hline & Gobioides broussonnetii & 2 & 0 & 0 & $\mathrm{EM}^{\mathrm{d}}$ & \\
\hline & Gobionellus oceanicus & 16 & 2 & 1 & $\mathrm{ES}^{\mathrm{a}, \mathrm{b}, \mathrm{e}}$ & $\mathrm{ZB}^{2,3}$ \\
\hline & Microgobius meeki & 78 & 22 & 5 & $\mathrm{MS}^{\mathrm{a}, \mathrm{e}}$ & $\mathrm{ZB}^{1}$ \\
\hline \multirow[t]{8}{*}{ Haemulidae } & Anisotremus surinamensis & 0 & 1 & 11008 & $\mathrm{MS}^{\mathrm{a}}$ & $\mathrm{ZB}^{1}$ \\
\hline & Boridia grossidens & 3 & 0 & 0 & & \\
\hline & Conodon nobilis & 0 & 0 & 899 & $\mathrm{MM}^{\mathrm{b}}$ & $\mathrm{ZB}^{3}$ \\
\hline & Genyatremus luteus & 6 & 0 & 24 & $\mathrm{MS}^{\mathrm{a}, \mathrm{b}, \mathrm{c}}$ & $\mathrm{ZB}^{2,3}$ \\
\hline & Haemulon steindachneri & 0 & 0 & 9 & $\mathrm{MM}^{\mathrm{a}}$ & $\mathrm{ZB}^{1,2}$ \\
\hline & Orthopristis ruber & 0 & 33 & 295 & MS & $\mathrm{ZB}^{1,12}$ \\
\hline & Pomadasys corvinaeformis & 0 & 6430 & 652 & $\mathrm{MM}^{\mathrm{a}, \mathrm{e}}$ & $\mathrm{ZB}^{1,2,10}$ \\
\hline & Pomadasys ramosus & 0 & 2 & 6 & MM & \\
\hline \multirow[t]{3}{*}{ Hemirhamphidae } & Hemiramphus brasiliensis & 0 & 18 & 209 & $\mathrm{MM}^{\mathrm{d}}$ & $\mathrm{PV}^{1}$ \\
\hline & Hyporhamphus robertii & 2 & 0 & 0 & MS & \\
\hline & Hyporhamphus unifasciatus & 203 & 47 & 1501 & $\mathrm{MS}^{\mathrm{a}, \mathrm{e}}$ & $\mathrm{OV}^{1,2}$ \\
\hline Lobotidae & Lobotes surinamensis & 0 & 6 & 1 & $\mathrm{MS}^{\mathrm{a}, \mathrm{d}}$ & $\mathrm{ZB}^{1}$ \\
\hline Lutjanidae & Lutjanus analis & 0 & 4 & 19 & $M^{d}$ & $\mathrm{PV}^{1}$ \\
\hline Monacanthidae & Stephanolepis hispidus & 13 & 69 & 96 & $\mathrm{ES}^{\mathrm{c}}$ & $\mathrm{ZB}^{1}$ \\
\hline \multirow[t]{5}{*}{ Mugilidae } & Mugil curema & 5 & 476 & 512 & $\mathrm{MM}^{\mathrm{a}, \mathrm{e}, \mathrm{f}}$ & $\mathrm{DV}^{2,26,27}$ \\
\hline & Mugil curvidens & 0 & 2 & 6 & $\mathrm{ES}^{\mathrm{d}}$ & $\mathrm{DV}^{1}$ \\
\hline & Mugil incilis & 0 & 0 & 7 & $\mathrm{EM}^{\mathrm{d}}$ & $\mathrm{DV}^{1}$ \\
\hline & Mugil liza & 2 & 365 & 563 & $\mathrm{CA}^{\mathrm{a}, \mathrm{d}}$ & $\mathrm{DV}^{1}$ \\
\hline & Mugil sp. & 219 & 181 & 528 & $\mathrm{MM}^{\mathrm{a}, \mathrm{e}}$ & DV \\
\hline Narcinidae & Narcine brasiliensis & 0 & 0 & 9 & $\mathrm{MS}^{\mathrm{a}}$ & $\mathrm{ZB}^{1}$ \\
\hline Ophichthidae & Ophichthus gomesii & 1 & 10 & 0 & MS & \\
\hline \multirow[t]{7}{*}{ Paralichthyidae } & Citharichthys arenaceus & 350 & 102 & 124 & $\mathrm{MS}^{\mathrm{c}, \mathrm{e}}$ & $\mathrm{ZB}^{1,10}$ \\
\hline & Citharichthys macrops & 0 & 0 & 1 & MS & $\mathrm{ZB}^{1}$ \\
\hline & Citharicthys spilopterus & 1 & 230 & 77 & $\mathrm{MS}^{\mathrm{a}, \mathrm{c}, \mathrm{e}}$ & $\mathrm{ZB}^{2,23,28}$ \\
\hline & Etropus crossotus & 6 & 131 & 743 & $\mathrm{ES}^{\mathrm{a}, \mathrm{c}}$ & $\mathrm{ZB}^{1,10}$ \\
\hline & Paralichthys brasiliensis & 0 & 0 & 3 & $\mathrm{MM}^{\mathrm{a}}$ & $\mathrm{ZB}^{2}$ \\
\hline & Paralichthys orbignyanus & 8 & 5 & 5 & $\mathrm{MM}^{\mathrm{e}}$ & $\mathrm{ZB}^{1}$ \\
\hline & Syacium papillosum & 0 & 0 & 1 & MS & $\mathrm{PV}^{1}$ \\
\hline Pleuronectidae & Oncopterus darwinii & 0 & 0 & 12 & MS & $\mathrm{ZB}^{1}$ \\
\hline Poeciliidae & Poecilia vivipara & 0 & 299 & 16 & $\mathrm{FM}^{\mathrm{d}}$ & $\mathrm{ZP}^{1}$ \\
\hline \multirow[t]{2}{*}{ Polynemidae } & Polydactylus oligodon & 0 & 0 & 1 & MS & $\mathrm{ZB}$ \\
\hline & Polidactylus virginicus & 0 & 11 & 82 & $\mathrm{MM}^{\mathrm{a}, \mathrm{b}, \mathrm{e}}$ & $\mathrm{ZB}^{1,2,3}$ \\
\hline Pomatomidae & Pomatomus saltatrix & 0 & 2 & 241 & $\mathrm{MS}^{\mathrm{e}}$ & $\mathrm{PV}^{1,29}$ \\
\hline Pristigasteridae & Pellona harroweri & 0 & 0 & 16 & $\mathrm{MS}^{\mathrm{a}}$ & $\mathrm{ZP}^{15}$ \\
\hline \multirow[t]{2}{*}{ Rhinobatidae } & Rhinobatos horkelii & 0 & 0 & 2 & $\mathrm{MS}^{\mathrm{a}, \mathrm{c}}$ & $\mathrm{ZB}^{1}$ \\
\hline & Rhinobatos percellens & 0 & 3 & 0 & MS & $\mathrm{ZB}^{1,30}$ \\
\hline Sciaenidae & Bairdiella ronchus & 201 & 692 & 57 & $\mathrm{ES}^{\mathrm{c}}$ & $\mathrm{ZB}^{1,2,31}$ \\
\hline & Ctenosciaena gracilicirrhus & 0 & 56 & 32 & MS & $\mathrm{ZB}^{1}$ \\
\hline & Cynoscion acoupa & 1 & 0 & 0 & $\mathrm{MM}^{\mathrm{a}, \mathrm{b}}$ & $\mathrm{ZB}^{3}$ \\
\hline & Cynoscion leiarchus & 0 & 8 & 49 & $\mathrm{MS}^{\mathrm{a}, \mathrm{c}, \mathrm{e}}$ & $\mathrm{PV}^{1,2,32}$ \\
\hline & Cynoscion microlepidotus & 0 & 0 & 4 & $\mathrm{ES}^{\mathrm{a}}$ & \\
\hline & Cynoscion striatus & 0 & 3 & 0 & $\mathrm{MS}^{\mathrm{d}}$ & $\mathrm{ZB}^{1}$ \\
\hline & Isopisthus parvipinnis & 0 & 1 & 15 & $\mathrm{MM}^{\mathrm{a}}$ & $\mathrm{ZB}^{1,33}$ \\
\hline & Larimus breviceps & 0 & 0 & 66 & $\mathrm{ES}^{\mathrm{a}}$ & $\mathrm{ZB}^{1,2,33}$ \\
\hline & Menticirrhus americanus & 2 & 117 & 1521 & $\mathrm{MM}^{\mathrm{a}, \mathrm{c}, \mathrm{d}, \mathrm{e}}$ & $\mathrm{ZB}^{1,10,32}$ \\
\hline & Menticirrhus littoralis & 0 & 18 & 1888 & $\mathrm{MM}$ & $\mathrm{ZB}^{2,10,12}$ \\
\hline & Micropogonias furnieri & 67 & 22 & 389 & $\mathrm{MM}^{\mathrm{a}, \mathrm{c}, \mathrm{e}}$ & $\mathrm{ZB}^{2,32,33}$ \\
\hline & Ophioscion punctatissimus & 0 & 0 & 22 & MS & $\mathrm{ZB}^{1,12}$ \\
\hline & Paralonchurus brasiliensis & 0 & 0 & 1 & MS & $\mathrm{ZB}^{1,33}$ \\
\hline & Pogonias cromis & 7 & 1 & 0 & MS & $\mathrm{ZB}^{1}$ \\
\hline & Stellifer brasiliensis & 5 & 3 & 3 & MM & $\mathrm{ZB}$ \\
\hline & Stellifer rastrifer & 19 & 137 & 1945 & $\mathrm{MM}^{\mathrm{a}, \mathrm{b}, \mathrm{c}, \mathrm{e}}$ & $\mathrm{ZB}^{3,34}$ \\
\hline & Stellifer stellifer & 4 & 17 & 3 & $\mathrm{ES}^{\mathrm{a}}$ & ZB \\
\hline
\end{tabular}




\begin{tabular}{|c|c|c|c|c|c|c|}
\hline & Umbrina canosai & 0 & 2 & 7 & MS & $\mathrm{ZB}^{1}$ \\
\hline & Umbrina coroides & 0 & 1 & 481 & MS & $\mathrm{ZB}^{1,12}$ \\
\hline \multirow[t]{2}{*}{ Scombridae } & Sarda sarda & 0 & 1 & 0 & & PV \\
\hline & Scomberomorus brasiliensis & 0 & 6 & 14 & $\mathrm{MS}^{\mathrm{a}}$ & $\mathrm{PV}^{1}$ \\
\hline Scorpaenidae & Scorpaena isthmensis & 0 & 1 & 0 & MS & PV \\
\hline \multirow[t]{5}{*}{ Serranidae } & Diplectrum radiale & 5 & 47 & 167 & $\mathrm{MS}^{\mathrm{a}, \mathrm{c}, \mathrm{e}}$ & $\mathrm{PV}^{1,2,15}$ \\
\hline & Hyporthodus nigritus & 0 & 0 & 1 & $\mathrm{MS}^{\mathrm{d}}$ & $\mathrm{ZB}^{1}$ \\
\hline & Mycteroperca bonaci & 0 & 0 & 1 & MS & $\mathrm{PV}^{1,2}$ \\
\hline & Mycteroperca rubra & 0 & 0 & 6 & MS & $\mathrm{ZB}^{1}$ \\
\hline & Rypticus randalli & 0 & 119 & 8 & $\mathrm{MS}^{\mathrm{a}}$ & $\mathrm{PV}^{9}$ \\
\hline \multirow[t]{2}{*}{ Sparidae } & $\begin{array}{l}\text { Archosargus } \\
\text { probatocephalus }\end{array}$ & 1 & 0 & 0 & MS & $\mathrm{ZB}^{1,2}$ \\
\hline & Archosargus rhomboidalis & 0 & 21 & 3 & $\mathrm{MM}^{\mathrm{a}}$ & $\mathrm{ZB}^{1,27}$ \\
\hline Sphyraenidae & Sphyraena tome & 0 & 1 & 2 & MM & PV \\
\hline Stromateidae & Peprilus paru & 0 & 0 & 25 & MS & $\mathrm{OV}^{1}$ \\
\hline \multirow[t]{5}{*}{ Syngnathidae } & Bryx dunckeri & 0 & 0 & 7 & $\mathrm{ES}^{\mathrm{d}}$ & $\mathrm{ZP}$ \\
\hline & Cosmocampus elucens & 0 & 1 & 3 & $\mathrm{MS}^{\mathrm{e}}$ & $\mathrm{ZP}$ \\
\hline & Hippocampus reidi & 1 & 2 & 9 & $\mathrm{ES}^{\mathrm{a}, \mathrm{c}}$ & $\mathrm{ZP}$ \\
\hline & Syngnathus folletti & 0 & 5 & 27 & $\mathrm{ES}^{\mathrm{e}}$ & $\mathrm{ZP}$ \\
\hline & Syngnathus pelagicus & 12 & 14 & 70 & $\mathrm{MS}^{\mathrm{e}}$ & $\mathrm{ZP}$ \\
\hline Synodontidae & Synodus foetens & 3 & 75 & 284 & $\mathrm{MS}^{\mathrm{a}, \mathrm{c}, \mathrm{e}}$ & $\mathrm{PV}^{1,9,15}$ \\
\hline \multirow[t]{3}{*}{ Tetraodontidae } & Lagocephalus laevigatus & 0 & 9 & 16 & $\mathrm{MM}^{\mathrm{d}, \mathrm{e}}$ & $\mathrm{ZB}^{1,2}$ \\
\hline & Sphoeroides greeleyi & 9 & 2575 & 494 & $\mathrm{ES}^{\mathrm{a}, \mathrm{d}, \mathrm{e}}$ & $\mathrm{ZB}^{1,9}$ \\
\hline & Sphoeroides testudineus & 1838 & 5849 & 433 & $\mathrm{ES}^{\mathrm{a}, \mathrm{c}, \mathrm{d}, \mathrm{e}}$ & $\mathrm{ZB}^{1,2,11}$ \\
\hline Trichiuridae & Trichiurus lepturus & 0 & 1 & 9 & $\mathrm{MS}^{\mathrm{a}, \mathrm{b}}$ & $\mathrm{PV}^{3}$ \\
\hline \multirow[t]{2}{*}{ Triglidae } & Prionotus nudigula & 0 & 3 & 24 & MS & $\mathrm{PV}^{1}$ \\
\hline & Prionotus punctatus & 0 & 10 & 206 & $\mathrm{MS}^{\mathrm{a}, \mathrm{c}, \mathrm{e}}$ & $\mathrm{ZB}^{1,2,35}$ \\
\hline \multirow[t]{2}{*}{ Uranoscopidae } & Astrocopus sexspinosus & 0 & 0 & 5 & & PV \\
\hline & Astrocopus u-graecum & 0 & 2 & 49 & $\mathrm{MS}^{\mathrm{e}}$ & $\mathrm{PV}^{1,36}$ \\
\hline
\end{tabular}

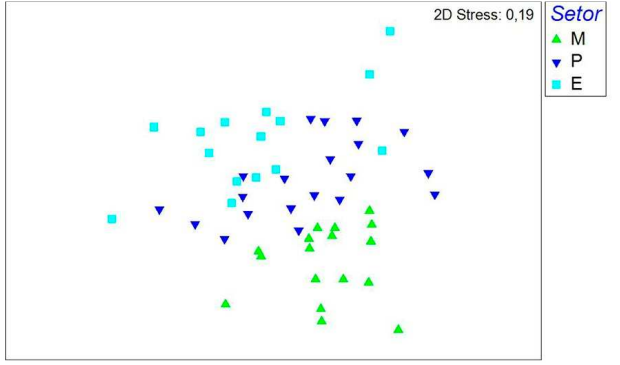

Fig. 2. Multidimensional ordination (MDS) based on the presence/absence of families between three salinity sectors ( $\mathrm{E}=$ euhaline; $\mathrm{P}=$ polyhaline; $\mathrm{M}=$ mesohaline).

Table 3. SIMPER results based on presence/absence of families within salinity sectors. Families most responsible for the mean similarity within each group and the dissimilarity between groups are shown in non-shaded and shaded boxes, respectively.

\begin{tabular}{|c|c|c|}
\hline Sectors & Mesohaline & Euhaline \\
\hline Mesohaline & $\begin{array}{l}\text { Atherinopsidae } \\
\text { Engraulidae } \\
\text { Gerreidae } \\
\text { Gobiidae }\end{array}$ & \\
\hline Euhaline & $\begin{array}{l}\text { Albulidae } \\
\text { Gobiidae } \\
\text { Ephippidae } \\
\text { Achiridae }\end{array}$ & $\begin{array}{l}\text { Carangidae } \\
\text { Clupeidae } \\
\text { Engraulidae } \\
\text { Tetraodontidae }\end{array}$ \\
\hline
\end{tabular}

The ordination of the sampling points based on the percentage of the estuarine use functional groups (Fig. 5), and respective ANOSIM, did not show clear separation between salinity sectors $(R$ Global $=0.183 ; P<0.01)$. Substantial overlap was observed between the mesohaline and euhaline sectors $(R=0.306 ; P<0.01)$, and even greater overlap was observed between the polyhaline and euhaline sectors $(R=0.236 ; P<0.01)$. There was no significant difference $(P>0.05)$ between the mesohaline and polyhaline sectors.

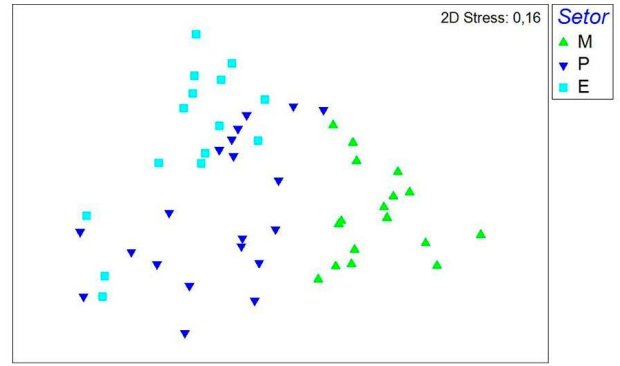

Fig. 3. Multidimensional ordination (MDS) based on the presence/absence of species between three salinity sectors $(\mathrm{E}=$ euhaline; $\mathrm{P}=$ polyhaline; $\mathrm{M}=$ mesohaline).

In the trophic functional composition percentage analysis (Fig. 6), the sectors sampled were not well defined in the nMDS; this lack of differentiation was confirmed by ANOSIM ( $R$-Global $=0.107 ; P<0.05)$. There were no significant differences among sectors in paired comparisons. 
Table 4. SIMPER results based on presence/absence of species within salinity sectors. Species most responsible for the mean similarity within each group and the dissimilarity between groups are shown in non-shaded and shaded boxes, respectively.

\begin{tabular}{|c|c|c|c|c|}
\hline $\begin{array}{l}\text { Sectors } \\
\text { Mesohaline }\end{array}$ & Mesohaline & Polyhaline & \multicolumn{2}{|c|}{ Euhaline } \\
\hline Mesohaline & $\begin{array}{l}\text { Anchoa } \\
\text { januaria } \\
\text { Atherinella } \\
\text { brasiliensis } \\
\text { Diapterus } \\
\text { rhombeus }\end{array}$ & & & \\
\hline Polyhaline & $\begin{array}{l}\text { Anchoa tricolor } \\
\text { Sphoeroides } \\
\text { greeleyi } \\
\text { Ctenogobius } \\
\text { shufeldti }\end{array}$ & $\begin{array}{l}\text { Atherinella } \\
\text { brasiliensis } \\
\text { Sphoeroides } \\
\text { testudineus } \\
\text { Anchoa tricolor }\end{array}$ & & \\
\hline Euhaline & $\begin{array}{l}\text { Trachinotus } \\
\text { falcatus } \\
\text { Harengula } \\
\text { clupeola } \\
\text { Trachinotus } \\
\text { carolinus }\end{array}$ & $\begin{array}{l}\text { Albula vulpes } \\
\text { Trachinotus } \\
\text { carolinus } \\
\text { Selene vomer }\end{array}$ & $\begin{array}{l}\text { Anc } \\
\text { tric } \\
\text { Har } \\
\text { clup } \\
\text { Tra } \\
\text { falc }\end{array}$ & $\begin{array}{l}\text { hoa } \\
\text { lor } \\
\text { engula } \\
\text { eola } \\
\text { chinotus } \\
\text { atus }\end{array}$ \\
\hline & ${ }_{\Delta}^{A}{ }_{\Delta}^{A}$ & 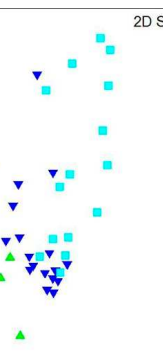 & & \begin{tabular}{|l|} 
setor \\
$\Delta \mathrm{M}$ \\
$\mathrm{P}$ \\
$\mathrm{P}$ \\
\end{tabular} \\
\hline
\end{tabular}

Fig. 4. Multidimensional ordination (MDS) based on the quantitative similarity of fish species between three salinity sectors $(\mathrm{E}=$ euhaline; $\mathrm{P}=$ polyhaline; $\mathrm{M}=$ mesohaline).

Table 5. SIMPER results based on the percentage of individuals within a species in each salinity sector. Species most responsible for the mean similarity within each group and the dissimilarity between groups are shown in nonshaded and shaded boxes, respectively.

\begin{tabular}{|c|c|c|c|}
\hline Sectors & Mesohaline & Polyhaline & Euhaline \\
\hline Mesohaline & $\begin{array}{l}\text { Anchoa } \\
\text { januaria } \\
\text { Atherinella } \\
\text { brasiliensis } \\
\text { Diapterus } \\
\text { rhombeus }\end{array}$ & & \\
\hline Polyhaline & $\begin{array}{l}\text { Atherinella } \\
\text { brasiliensis } \\
\text { Anchoa } \\
\text { januaria } \\
\text { Diapterus } \\
\text { rhombeus }\end{array}$ & $\begin{array}{l}\text { Atherinella } \\
\text { brasiliensis } \\
\text { Sphoeroides } \\
\text { greeleyi } \\
\text { Anchoa tricolor }\end{array}$ & \\
\hline Euhaline & $\begin{array}{l}\text { Anchoa } \\
\text { januaria } \\
\text { Atherinella } \\
\text { brasiliensis } \\
\text { Harengula } \\
\text { clupeola }\end{array}$ & $\begin{array}{l}\text { Atherinella } \\
\text { brasiliensis } \\
\text { Harengula } \\
\text { clupeola } \\
\text { Anchoa tricolor }\end{array}$ & $\begin{array}{l}\text { Atherinella } \\
\text { brasiliensis } \\
\text { Harengula } \\
\text { clupeola } \\
\text { Anchoa } \\
\text { tricolor }\end{array}$ \\
\hline
\end{tabular}

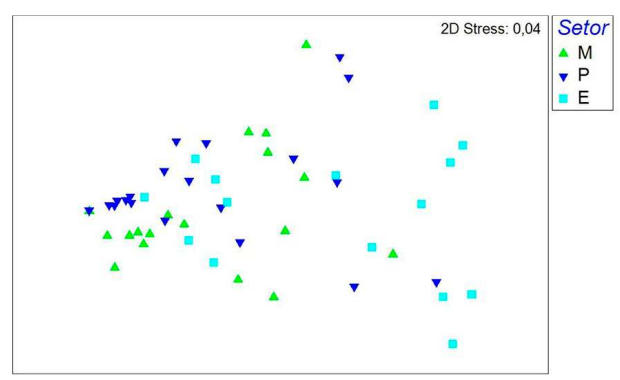

Fig. 5. Multidimensional ordination (MDS) based on the percentage of fish within estuarine use guilds between three salinity sectors $(\mathrm{E}=$ euhaline; $\mathrm{P}=$ polyhaline; $\mathrm{M}=$ mesohaline).

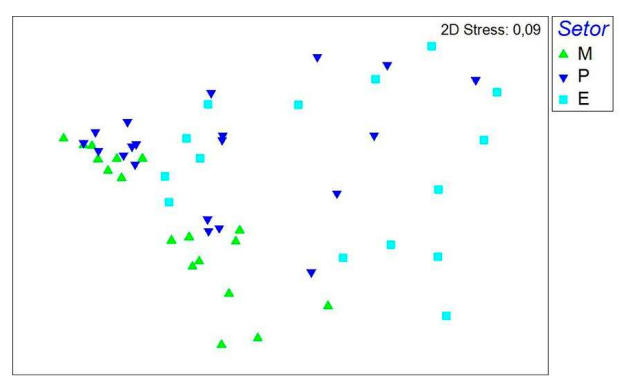

Fig. 6. Multidimensional ordination (MDS) based on the percentage of fish within trophic guilds between three salinity sectors $(\mathrm{E}=$ euhaline; $\mathrm{P}=$ polyhaline; $\mathrm{M}=$ mesohaline)

\section{Discussion}

In this study we tested the effect of the eastwest salinity gradient of the PEC on the structure of shallow water fish fauna, described in taxonomic (families and species) and functional terms. We found that the taxonomic metrics were better able to characterize fish assemblages along the estuarine salinity gradient than were functional metrics. In other words, the osmoregulatory abilities of the various species played a greater role in determining the spatial distribution of an assemblage than did their functional traits. We used a mean spatial model that represented an individual's salinity affinity well. However, individuals of all functional groups occurred in all salinity sectors and, thus, this metric was not useful for differentiating assemblages along the salinity gradient using a mean model. Our results differed from those of other studies, which emphasized the importance of functional groups in determining fish assemblages along salinity gradients (ELLIOTT; DEWAILLY, 1995; MATHIESON et al., 2000; CHAVES; BOUCHEREAU, 2004; HARRISON; WHITFIELD, 2012). 
Among the taxonomic metrics employed here, presence/absence of families was less efficient than species in discriminating assemblages across sectors. In fact, each family may contain many species, each with a different capacity for reaching their optima in different salinity zones (ELLIOTT et al., 2007, BLABER, 2008). For example, $E$. melanopterus and E. argenteus were two of the most important species in the PEC mesohaline and polyhaline zones, respectively (SANTOS et al., 2002; VENDEL et al., 2002, 2003; SPACH et al., 2006; FALCÃO et al., 2006; FÉLIX et al., 2006; OLIVEIRA-NETO et al., 2008, CONTENTE et al., 2011a).

Atherinella brasiliensis, Anchoa januaria, Diapterus rhombeus and Harengula clupeola were the most abundant species, both along the entire east-west axis of the PEC and within each of the sectors individually. A. brasiliensis was the most abundant fish throughout the entire east-west axis, but showed a tendency for larger abundance within inner estuarine zones. These fish are generalists and opportunistic estuarine omnivores, tolerant of any estuarine environmental conditions (CONTENTE et al., 2011b). The wide range and dominance of this species in the PEC, as well as in other southern Brazilian estuaries, are primarily due to these plastic trophic features and a fast growing, short-lived life cycle (CONTENTE et al., 2011a). The species generally inhabits shallow bays and estuaries and can form large populations [as also in other subtropical estuaries, e.g., Lagoa do Patos Estuary; (GARCIA et al., 2001)], thus comprising a key component of the trophic web (CONTENTE et al., 2011b). A. januaria and D. rhombeus were more characteristic of the mesohaline sector of the PEC. A. januaria forms large schools and is associated with the internal areas of bays and estuaries; it is influenced by continental drainage and less saline waters. Juveniles of $D$. rhombeus prefer shallow mesohaline areas, such as beaches and mangrove channels, to grow and store somatic reserves during the reproductive period (MENEZES; FIGUEIREDO, 1980; CHAVES; OTTO, 1998). H. clupeola (zooplanktivorous marine straggler) and A. tricolor (zooplanktivorous estuarine species) were more abundant in the euhaline sector a finding was not in accord with observations from other estuaries (FALCÃO et al., 2006; FÉLIX et al., 2006; OLIVEIRA-NETO et al., 2008; CONTENTE et al., 2011a). Differences in sector usage by A. januaria, $D$. rhombeus and $H$. clupeola most likely occur due to differences in the salinity tolerances of these species. Despite being euryhaline, estuarine species generally reach their optima within specific salinity ranges (PAPERNO et al., 2001; PAPERNO; BRODIE, 2004; CONTENTE et al., 2011a) due to strong speciesspecific physiological responses to the estuarine salinity gradient (BLABER, 2008).
There were some differences in sampling effort and net mesh size given in the studies from which data were drawn for the assemblage analyses. Nevertheless, samples were representative of the spatial pattern of shallow water fish fauna, because the predominance of seine nets reduced potential bias (ELLIOTT; DEWAILLY, 1995). Analyses were limited to the fauna captured with seine and capéchades nets; thus our conclusions were limited to shallow water fish fauna consisting of individuals with mean total lengths of less than $100 \mathrm{~mm}$. Our conclusions must be tested using other components of the PEC fish fauna (e.g., larger fishes from the main channel and open water environments). Another potential source of error is that the same species could fit into different guilds according to the site and region due to differences in the availability of resources and environmental conditions (ELLIOTT et al., 2007; BARLETTA; BLABER, 2007). For example, in our study $D$. rhombeus was classified as an estuarine species, while in Vilar et al. (2011) it was considered a marine migrant. Similarly, G. luteus was classified here as a marine straggler, while Barletta and Blaber (2007) considered it to be an estuarine species. These discrepancies highlight the need for more comprehensive information on the ecology and biology of the species inhabiting SW Atlantic estuaries (BARLETTA et al., 2010) to resolve such ambiguities.

Although many studies of Brazilian estuaries have classified species into functional guilds (VIEIRA; MUSICK, 1993; GARCIA; VIEIRA, 2001; CHAVES; BOUCHEREAU, 2004; ANDRADETUBINO et al., 2008; REIS-FILHO et al., 2010; VILAR et al., 2011), they have not tested the effect of the estuarine salinity gradient on the functional traits of the assemblages. We found that traditional taxonomic metrics were a better indicator of structural changes than were trophic groups or membership in categories of estuarine use. We have demonstrated that the salinity gradient has a key impact on the taxonomic structure of PEC fish assemblages because of the consistent link between estuarine fish fauna along a longitudinal gradient and the longitudinal salinity gradient of the estuary; this type of impact is a general feature among estuaries worldwide (BULGER et al., 1993; JAUREGUIZAR et al., 2004; AKIN et al., 2005; CHAGAS et al., 2006; BARLETTA; BLABER, 2007; BARLETTA et al., 2008; VILAR et al., 2011).

We suggest that future studies consider environments on a smaller spatial scale along the eastwest axis of the PEC. The similar results for the polyhaline and euhaline sectors may have been due to their proximity to the Cotinga sub-estuary (located between the euhaline and polyhaline sectors) which possesses different morphological and hydrological 
characteristics (primarily related to the tides and the energy in the drainage area) from the east-west axis of the PEC (NOERNBERG et al., 2006). This environment is composed of winding rivers and extensive floodplains, which can form unvegetated sandbanks and confer unique characteristics to this area. In addition, this sub-estuary is near the mixing zone of the estuary. We had expected that these habitat features would have had a greater influence on the trophic features of the fish fauna than the long salinity gradient examined in this study. A similar relationship might be expected for the Nhundiaquara and Cachoeira sub-estuaries in the mesohaline sector.

\section{ACKNOWLEDGMENTS}

We would like to thank the CEM Geological Oceanography Laboratory for the map data base and Pâmela Emanuelly Cattani for her valuable help with the map. We would like to thank the "Fundação Grupo Boticário de Proteção à Natureza" for financial support. Funding was provided by the CAPES foundation through a master's grant to Ana Carolina dos Passos.

\section{REFERENCES}

AKIN, S.; BUHAN, E.; WINEMILLER, K. O.; YILMAZ, H. Fish assemblage structure of Koycegiz Lagoon - Estuary, Turkey: spatial and temporal distribution patterns in relation to environmental variation. Estuarine, Coastal Shelf Sci., v. 64, n. 4, p. 671-684, 2005.

ALVES, M. I. M.; SOARES FILHO, A. A. Peixes do estuário do Rio Jaguaribe (Ceará - Brasil): aspectos fisioecológicos. Cienc. Agron., v. 27, n. 1/2, p. 5-16, 1996.

ANDRADE-TUBINO, M. F.; RIBEIRO, A. L. R.; VIANNA, M. Organização espaço-temporal das ictiocenoses demersais nos ecossistemas estuarinos brasileiros: uma síntese. Oecol. Bras., v. 12, n. 4, p. 640-661, 2008.

ANGEL, A.; OJEDA, F. P. Structure and trophic organization of subtidal fish assemblages on the northern Chilean coast: the effect of habitat complexity. Mar. Ecol.: Prog. Ser., v. 217, p. 81-91, 2001.

ARAÚJO, F. G. Hábitos alimentares de três bagres marinhos (Ariidae) no estuário da Lagoa dos Patos (RS), Brasil. Rev. Atlântica, v. 7, p. 47-63, 1984.

BARLETTA, M.; BLABER, S. J. M. Comparison of fish assemblages and guilds in tropical habitats of the Embley (Indo-West Pacific) and Caeté (Western Atlantic) estuaries. Bull. Mar. Sci., v. 80, n. 3, p. 647-680, 2007.

BARLETTA, M.; AMARAL, C. S.; CORRÊA, M. F. M.; GUEBERT, F.; DANTAS, D. V.; LORENZI, L.; SAINT-PAUL, U. Factors affecting seasonal variations in demersal fish assemblages at an ecocline in a tropicalsubtropical estuary. J. Fish Biol., v. 73, n. 3, p. 13141336, 2008.

BARLETTA, M.; JAUREGUIZAR, A. J.; BAIGUN, C.; FONTOURA, N. F.; AGOSTINHO, A. A.; ALMEIDAVAL, V. M. F.; VAL, A. L.; TORRES, R. A.
JIMENES-SEGURA, L. F.; GIARRIZZO, T.; FABRÉ, N. N.; BATISTA, V. S.; LASSO, C.; TAPHORN, D. C.; COSTA, M. F.; CHAVES, P. T.; VIEIRA, J. P.; CORRÊA, M. F. M. Fish and aquatic habitat conservation in South America: a continental overview with emphasis on neotropical systems. J. Fish Biol., v. 76, n. 9, p. 2118-2176, 2010.

BLABER, S. J. M. Tropical estuarine fishes: ecology, exploration and conservation. Oxford: WileyBlackwell Science, 2008. 384p. (Fish and aquatic resources series; 7).

BLONDEL, J. Guilds or functional groups: does it matter? Oikos, v. 100, n. 2, p. 223-231, 2003.

BORTOLUZZI, T.; ASCHENBRENNER, A. C.; SILVEIRA, C. R.; ROOS, D. C.; LEPKOSKI, E. D.; MARTINS, J. A.; GOULART, M. G.; QUEROL, E.; QUEROL, M. V. Hábito alimentar da sardinha prata, Lycengraulis grossidens (Spix \& Agassiz, 1829), (Pices, Engraulidae), Rio Uruguai médio, sudoeste do Rio Grande do Sul, Brasil. Biodiversidade Pampeana, v. 4, n. 1, p. 11-23, 2006.

BULGER, A. J.; HAYDEN, B. P.; MONACO, M. E.; NELSON, D. M.; MCCORMICK-RAY, M. G. Biologically-based estuarine salinity zones derived from a multivariate analysis. Estuaries, v. 16, n. 2, p. 311322, 1993.

CAIRES, R. A.; PICHLER, H. A.; SPACH, H. L.; IGNÁCIO, J. M. Opsanus brasiliensis Rotundo, Spinelli \& Zavalla-Camin, 2005 (Teleostei: Batrachoidiformes: Batrachoididae), sinônimo-júnior de Opsanus beta (Goode \& Bean, 1880), com notas sobre a ocorrência da espécie na costa brasileira. Biota Neotrop., v. 7, n. 2, p. 135-139, 2007.

CARVALHO-FILHO, A.; SANTOS, S.; SAMPAIO, I. Macrodon atricauda (Günther, 1880) (Perciformes: Sciaenidae), a valid species from the southwestern Atlantic, with comments on its conservation. Zootaxa, v. 2519 , p. 48-58, 2010.

CASSEMIRO, F. A. S.; HAHN, N. S.; RANGEL, T. F. L. V. B. Diet and trophic ecomorphology of the silverside, Odontesthes bonariensis, of the Salto Caxias reservoir, Rio Iguaçu, Paraná, Brazil. Neotrop. Ichthyol., v. 1, n. 2, p. 127-131, 2003

CASTILLO-RIVERA, M.; KOBELKOWSKY, A; CHÁVEZ, A. M. Feeding biology of the flatfish Citharichthys spilopterus (Bothidae) in a tropical estuary of Mexico. J. Appl. Ichthyol., v. 16, n. 2, p. 73-78, 2000.

CERVIGÓN MARCOS, F. Los peces marinos de Venezuela. Caracas: Fundación Científica Los Roques, 1994. v.3.

CHAGAS, L. P.; JOYEUX, J. -C.; FONSECA, F. R. Smallscale spatial changes in estuarine fish: subtidal assemblages in tropical Brazil. J. Mar. Biol. Assoc. U. K., v. 86, n. 4, p. 861-875, 2006.

CHAVES, P. T. C.; OTTO, G. Aspectos biológicos de Diapterus rhombeus (Cuvier) (Teleostei, Gerreidae) na Baía de Guaratuba, Paraná, Brasil. Rev. Bras. Zool., v. 15, n. 2, p. 289-295, 1998

CHAVES, P. T.; VENDEL, A. L. Feeding habits of Stellifer rastrifer (Perciformes, Sciaenidae) at Guaratuba mangrove, Paraná, Brazil. Braz. Arch. Biol. Technol., v. 41, n. 4, p. 423-428, 1998. 
CHAVES, P. T.; UMBRIA, S. C. Changes in the diet composition of transitory fishes in coastal systems, estuary and continental shelf. Braz. Arch. Biol. Technol., v. 46, n. 1, p. 41-46, 2003.

CHAVES, P.; BOUCHEREAU, J. -L. Trophic organization and functioning of fish populations in the Bay of Guaratuba, Brazil, on the basis of a trophic contribution factor. Acta Adriat., v. 45, n. 1, p. 83-94, 2004.

CLARKE, K. R.; WARWICK, R. M. Changes in marine communities: an approach to statistical analysis and interpretation. 2. ed. Plymouth: Plymouth Marine Laboratory: Primer-e, 2001. 176p.

CLARKE，K. R.; GORLEY, R. N. PRIMER v6: user manual/tutorial. Plymouth: PRIMER-E, 2006. 91p.

CONTENTE, R. F. Partição inter-específica e efeitos sazonais, espaciais e ontogenéticos no uso de recursos tróficos por seis teleostei em um sistema estuarino sub-tropical. 180 p. Dissertação (Mestrado em Ciências Biológicas) - Universidade Federal do Paraná, Curitiba, 2008 .

CONTENTE, R. F.; STEFANONI, M. F.; GADIG, O. B. F. Size-related shifts in dietary composition of Centropomus parallelus (Perciformes: Centropomidae) in an estuarine ecosystem of the southeastern coast of Brazil. J. Appl. Ichthyol., v. 25, n. 3, p. 335-342, 2009.

CONTENTE, R. F.; STEFANONI, M. F.; SPACH, H. L. Fish assemblage structure in an estuary of the Atlantic Forest biodiversity hotspot (southern Brazil). Ichthyol. Res., v. 58, n. 1, p. 38-50, 2011a.

CONTENTE, R. F.; STEFANONI, M. F.; SPACH, H. L. Feeding ecology of the Brazilian silverside Atherinella brasiliensis (Atherinopsidae) in a sub-tropical estuarine ecosystem. J. Mar. Biol. Assoc. U. K., v. 91, n. 6, p. 1197-1205, 2011b

CORRÊA, M. O. D. A.; UIEDA, V. S. Diet of the ichthyofauna associated with marginal vegetation of a mangrove forest in southeastern Brazil. Iheringia, Ser. Zool., v. 97, n. 4, p. 486-497, 2007.

CRAIG, M. T.; HASTINGS, P. A. A molecular phylogeny of the groupers of the subfamily Epinephelinae (Serranidae) with a revised classification of Epinephelini. Ichthyol. Res., v. 54, n. 1, p. 1-17, 2007.

ELLIOTT, M.; DEWAILLY, F. The structure and components of European estuarine fish assemblages. Neth. J. Aquat. Ecol., v.29, v. 3/4, p. 397-417, 1995.

ELLIOTT, M.; WHITFIELD, A. K.; POTTER, I. C. BLABER, S. J. M.; CYRUS, D. P.; NORDLIE, F. G.; HARRISON, T. D. The guild approach to categorizing estuarine fish assemblages: a global review. Fish Fish., v. 8, n. 3 , p. 241-268, 2007.

ESCHMEYER, W. N. (Ed.). Catalog of fishes. California Academy of Sciences, San Francisco. Available in: http://www.calacademy.org/research/ichthyology/catalog /. Accessed in: Oct. 20, 2010.

FALCÃO, M. G.; SARPÉDONTI, V.; SPACH, H. L.; OTERO, M. E. B.; QUEIROZ, G. M. L. N.; SANTOS, C. A ictiofauna em planícies de maré das Baías de Laranjeiras e de Paranaguá, Paraná, Brasil. Rev. Bras. Zoocienc., v.8, n. 2, p. 125-138, 2006.

FALCÃO, M. G.; PICHLER, H. A.; FÉLIX, F. C.; SPACH, H. L.; BARRIL, M. E.; ARAÚJO, K. C. B.; GODEFROID, R. S. A ictiofauna como indicador de qualidade ambiental em planícies de maré do Complexo
Estuarino de Paranaguá, Brasil. Cad. Esc. Saúde Unibrasil, v. 1, p. 1-16, 2008.

FÉLIX, F. C.; SPACH, H. L.; HACKRADT, C. W.; MORO, P. S.; ROCHA, D. C. Abundância sazonal e a composição da assembléia de peixes em duas praias estuarinas da Baía de Paranaguá, Paraná. Rev. Bras. Zoocienc., v. 8, n. 1, p. 35-47, 2006.

FÉLIX, F. C.; SPACH, H. L.; MORO, P. S.; HACKRADT, C. W.; QUEIROZ, G. M. L. N.; HOSTIM-SILVA, M. Ichthyofauna composition across a wave: energy gradient on Southern Brazil beaches. Braz. J. Oceanogr., v. 55, n. 4, p. 281-292, 2007a.

FÉLIX, F. C.; SPACH, H. L.; MORO, P. S.; SCHWARZ Jr., R.; SANTOS, C.; HACKRADT, C. W.; HOSTIMSILVA, M. Utilization patterns of surf zone inhabiting fish from beaches in Southern Brazil. PanamJAS, v. 2, n. 1, p. 27-39, 2007 b.

FIGUEIREDO, J. L. Manual de peixes marinhos do Sudeste do Brasil. São Paulo: Universidade de São Paulo, Museu de Zoologia, 1977. 104 p. [I. Introdução. Cações, raias e quimeras].

FIGUEIREDO, J. L.; MENEZES, N. A. Manual de peixes marinhos do sudeste do Brasil. II. Teleostei (1). São Paulo: Universidade de São Paulo, Museu de Zoologia, 1978. 110p.

FIGUEIREDO, J. L.; SALLES, A. C. R.; RABELO, L. B. Sardinella brasiliensis (Steindachner, 1879) (Teleostei: Clupeidae), nome válido aplicado à sardinha verdadeira no sudeste do Brasil. Pap. Avulsos Dep. Zool., Secr. Agric., Ind. Comer., v. 50, n. 18, p. 281-283, 2010.

FRANCO, A.; FRANZOI, P.; TORRICELLI, P. Structure and functioning of Mediterranean Lagoon fish assemblages: a key for the identification of water body types. Estuarine, Coastal Shelf Sci., v. 79, n. 3, p. 549558, 2008.

FROESE, R.; PAULY, D. FishBase. Version (07/2010). Available in: www.fishbase.org Accessed in: Oct. 27, 2010.

GARCIA, A. M.; VIEIRA, J. P. O aumento da diversidade de peixes no estuário da Lagoa dos Patos durante o episódio El Niño 1997-1998. Rev. Atlântica, v. 23, p. 133-152, 2001

GARCIA, A. M.; VIEIRA, J.P.; WINEMILLER, K.O. Dynamics of the shallow-water fish assemblage of the Patos Lagoon estuary (Brazil) during cold and warm ENSO episodes. J. Fish Biol., v. 59, n. 5, p. 1218-1238, 2001

GARRISON, L. P.; LINK, J. S. Dietary guild structure of fish community in the Northeast United States continental shelf ecosystem. Mar. Ecol.: Prog. Ser., v. 202, p. 231240, 2000.

GODEFROID, R. S.; HOFSTAETTER, M.; SPACH, H. L. Structure of the fish assemblage in the surf zone of the beach at Pontal do Sul, Paraná. Nerítica, v. 11, p. 77-93, 1997.

GODEFROID, R. S.; SPACH, H. L.; SCHWARZ Jr., R.; QUEIROZ, G. M. L. N.; OLIVEIRA NETO, J. F. Efeito da lua e da maré na captura de peixes em uma planície de maré da Baía de Paranaguá, Paraná, Brasil. Bol. Inst. Pesca, v. 29, n. 1, p. 47-55, 2003.

GREGG, J. C.; FLEEGER, J. W. Importance of emerged and suspended meiofauna to the diet of the darter goby (Gobionellus boleosoma Jordan and Gilbert). J. Exp. Mar. Biol. Ecol., v. 209, n. 1/2, p. 123-142, 1997. 
GUEDES, A. P. P.; ARAÚJO, F. G. Trophic resource partitioning among five flatfish species (Actinopterygii, Pleuronectiformes) in a tropical bay in south-eastern Brazil. J. Fish Biol., v. 72, n. 4, p. 10351054, 2008.

HACKRADT, C. W.; FÉLIX-HACKRADT, F. C.; PICHLER, H. A.; SPACH, H. L.; SANTOS, L. O. Factors influencing spatial patterns of the ichthyofauna of low energy estuarine beaches in southern Brazil. J. Mar. Biol. Assoc. U. K., v. 91, n. 6, p. $1345-$ 1357, 2011.

HARRISON, T. D.; WHITFIELD, A. K. Fish trophic structure in estuaries, with particular emphasis on estuarine typology and zoogeography. J. Fish Biol., v. 81, n. 6, p. 2005-2029, 2012.

HIATT, R. W.; STRASBURG, D. W. Ecological relationships of the fish fauna on coral reefs of the Marshall Islands. Ecol. Monogr., v. 30, n. 1, p. 66-127, 1960.

IGNÁCIO, J. M.; SPACH, H. L. Variação entre o dia e a noite nas características da ictiofauna da infralitoral raso do Maciel, Baía de Paranaguá, Paraná. Rev. Bras. Zoocienc., v.11, n. 1, p. 25-37, 2009.

JAUREGUIZAR, A. J.; MENNI, R.; GUERREIRO, R.; LASTA, C. Environmental factors structuring fish communities of the Rio de la Plata estuary. Fish. Res., v. 66, n. 2/3, p. 195-211, 2004.

KNOPPERS, B. A.; BRANDINI, F. P.; THAMM, C. A. Ecological studies in the Bay of Paranaguá. II. Some physical and chemical characteristics. Nerítica, v. 2, n. 1, p. 1-36, 1987.

LANA, P. C.; MARONE, E.; LOPES, R. M.; MACHADO, E. C. The Subtropical Estuarine Complex of Paranaguá Bay, Brazil, In: SEELIGER, U.; KJERFVE, B. (Eds.). Coastal marine ecosystems of Latin America. Berlin: New York: Springer-Verlag, 2001. p. 131-145. (Ecological studies; v. 144).

LOBRY, J.; MOURAND, L.; ROCHARD, E.; ELIE, P. Structure of the Gironde estuarine fish assemblages: a comparison of European estuaries perspective. Aquat. Living Resour., v. 16, n. 2, p. 47-58, 2003.

MARONE, E.; MACHADO, E. C.; LOPES, R. M.; SILVA, E. T. Land-ocean fluxes in the Paranagua Bay estuarine system, Southern Brazil. Braz. J. Oceanogr., v. 53, n. 3/4, p. 169-181, 2005.

MATHIESON, S.; CATTRIJSSE, A.; COSTA, M. J.; DRAKE, P.; ELLIOTT, M.; GARDNER, J.; MARCHAND, J. Fish assemblages of European tidal marshes: a comparison based on species, families and functional guilds. Mar. Ecol.: Prog. Ser., v. 204, p. 225242, 2000

MENEZES, N. A.; FIGUEIREDO, J. L. Manual de peixes marinhos do sudeste do Brasil. IV. Teleostei (3). São Paulo: Universidade de São Paulo, Museu de Zoologia, 1980. 96p.

MENEZES, N. A.; BUCKUP, P. A.; FIGUEIREDO, J. L.; MOURA, R. L. (Eds.). Catálogo de espécies de peixes marinhos do Brasil. São Paulo: Universidade de São Paulo, Museu de Zoologia, 2003. 160 p.

NAGELKERKEN, I.; VAN DER VELDE, G.; MORINIÈRE, E. C. D. L. Fish feeding guilds along a gradient of bay biotopes and coral reef depth zones. Aquat. Ecol., v. 35, n. 1, p. 73-86, 2001.
NETTO, S. A.; LANA, P. C. Benthic macrofauna of Spartina alterniflora marshes and nearby unvegetated tidal flats of Paranagua Bay (SE Brazil). Nerítica, v. 10, n. 1/2, p. 41$55,1996$.

NETTO, S. A.; LANA, P. C. Influence of Spartina alterniflora on superficial sediment characteristics of tidal flats in Paranagua Bay (south-eastern Brazil). Estuarine, Coastal Shelf Sci., v. 44, n. 5, p. 641-648, 1997.

NOERNBERG, M. A.; LAUTERT, L. F. C.; ARAÚJO, A. D.; MARONE, E.; ANGELOTTI, R.; NETTO Jr., J. P. B.; KRUG, L. A. Remote sensing and GIS integration for modelling the Paranagua Estuarine Complex - Brazil. J. Coastal Res., v. SI39, p. 1627-1631, 2006.

OLIVEIRA-NETO, J. F.; SPACH, H. L.; SCHWARZ Jr., R.; PICHLER, H. A. Diel variation in fish assemblages in tidal creeks in southern Brazil. Braz. J. Biol., v. 68, n. 1, p. 37-43, 2008.

PAPERNO, R.; MILLE, K. J.; KADISON, E. Patterns in species composition of fish and selected invertebrate Assemblages in Estuarine Subregions near Ponce de Leon inlet, Florida. Estuarine, Coastal Shelf Sci., v. 52, n. 1, p. 117-130, 2001.

PAPERNO, R.; BRODIE, R. B. Effects of environmental variables upon the spatial and temporal structure of a fish community in a small, freshwater tributary of the Indian River Lagoon, Florida. Estuarine, Coastal Shelf Sci., v. 61, n. 2, p. 229-241, 2004.

PASSOS, A. C.; CONTENTE, R. F.; ARAÚJO, C. C. V.; DAROS, F. A. L. M.; SPACH, H. L.; ABILHÔA, V.; FÁVARO, L. F. Fishes of Paranagua Estuarine Complex, South West Atlantic. Biota Neotrop., v. 12, n. 3, p. 226-238, 2012.

PIEDRAS, S. R. N.; POUEY, J. L. O. Alimentação do peixerei (Odontesthes bonariensis, Atherinopsidae) nas lagoas Mirim e Mangueira, Rio Grande do Sul, Brasil. Iheringia, Ser. Zool., v. 95, n. 2, p. 117-120, 2005.

RANDALL, J. E. Food habits of reef fishes of the West Indies. Coral Gables: Institute of Marine Sciences, University of Miami, 1967. p. 665-847. (Studies in Tropical Oceanography; 5).

REIS-FILHO, J. A.; NUNES, J. A. C. D.; FERREIRA, A. Estuarine ichthyofauna of the Paraguaçu River, Todos os Santos Bay, Bahia, Brazil. Biota Neotrop., v. 10, n. 4, p. 301-312, 2010.

SANTOS, C.; SCHWARZ Jr., R.; OLIVEIRA NETO, J. F.; SPACH, H. L. A ictiofauna em duas planícies de maré do setor euhalino da Baía de Paranaguá, Paraná. Bol. Inst. Pesca, v. 28, n. 1, p. 49-60, 2002.

SACCARDO, S. A.; ROSSI-WONGTSCHOWSKI, C. L. D. B. Biologia e avaliação do estoque da Sardinha Sardinella brasiliensis: uma compilação. Rev. Atlântica, v. 13 , n. 1, p. 29-43, 1991.

SELLESLAGH, J.; AMARA, R.; LAFFARGUE, P.; LESOURD, S.; LEPAGE, M.; GIRARDIN, M. Fish composition and assemblage structure in three Eastern English Channel macrotidal estuaries: a comparison with other French estuaries. Estuarine, Coastal Shelf Sci., v. 81, n. 2, p. 149-159, 2009.

SERGIPENSE, S.; CARAMASHI, E. P.; SAZIMA, I. Morfologia e hábitos alimentares de duas espécies de Engraulidae (Teleostei-Clupeiformes) na Baía de Sepetiba, Rio de Janeiro. Rev. Bras. Oceanogr., v. 47, n. 2, p. 173-188, 1999. 
SHEAVES, M. J. Scale-dependent variation in composition of fish fauna among sandy tropical estuarine embayments. Mar. Ecol.: Prog. Ser., v. 310, p. 173-184, 2006.

SHEAVES, W. L.; CRAIG, M. T. Casting the Percomorph net widely: the importance of broad taxonomic sampling in the search for the placement of the serranid and percid fishes. Copeia, v. 2007, n. 1, p. 35-55, 2007. (Faltou acrescentar este autor no texto)

SOARES, L. S. H.; VAZZOLER, A. E. A. M. Diel changes in food and feeding activity of sciaenid fishes from the South Western Atlantic, Brazil. Braz. J. Biol., v. 61, n. 2, 197-216, 2001.

SPACH, H. L.; SANTOS, C.; GODEFROID, R. S. Padrões temporais na assembléia de peixes na gamboa do Sucuriú, Baía de Paranaguá, Brasil. Rev. Bras. Zool., v. 20, n. 4, p. 591-600, 2003.

SPACH, H. L.; GODEFROID, R. S.; SANTOS, C.; SCHWARZ Jr., R.; QUEIROZ, G. M. L. N. Temporal variation in fish assemblage composition on a tidal flat. Braz. J. Oceanogr., v. 52, n. 1, p. 47-58, 2004a.

SPACH, H. L.; SANTOS, C.; GODEFROID, R. S.; NARDI, M.; CUNHA, F. A study of the fish community structure in a tidal creek. Braz. J. Biol., v. 64, n. 2, p. 337-351, $2004 b$.

SPACH, H. L.; FÉLIX, F. C.; HACKRADT, C. W. LAUFER, D. C.; MORO, O. S.; CATTANI, A. P. Utilização de ambientes rasos por peixes na baía de Antonina, Paraná. Biociências, v. 14, n. 2, p. 125-135, 2006.

STEFANONI, M. F. Ictiofauna e ecologia trófica de peixes em ambientes praiais da Ilha das Peças, Complexo Estuarino de Paranaguá, Paraná. 2008. 143p. Dissertação (Mestrado) - Universidade Federal do Paraná, Curitiba, 2007.

TEIXEIRA, R. L.; HAIMOVICI, M. Distribuição, reprodução e hábitos alimentares de Prionotus punctatus e $P$. nudigula (Pices: Triglidae) no litoral do Rio Grande do Sul, Brasil. Rev. Atlântica, v. 11, p. 13-45, 1989.

TEIXEIRA, R. L. Distribution and feeding habits of the young common snook, Centropomus undecimalis (Pisces: Centropomidae), in the shallow waters of a tropical Brazilian estuary. Bol. Mus. Biol. Mello Leitão, v. 6 , p. $35-46,1997$.
VENDEL, A. L.; CHAVES, P. T. C. Alimentação de Bairdiella ronchus (Cuvier) (Teleostei, Sciaenidae) na Baía de Guaratuba, Paraná, Brasil. Rev. Bras. Zool., v. 15, n. 2, p. 297-305, 1998.

VENDEL, A. L.; SPACH, H. L.; LOPES, S. G.; SANTOS, C. Structure and dynamics of fish assemblages in a tidal creek environment. Braz. Arch. Biol. Technol., v. 45, n. 3, p. 365-373, 2002.

VENDEL, A. L.; LOPES, S. G.; SANTOS, C.; SPACH, H. L. Fish assemblages in a tidal flat. Braz. Arch. Biol. Technol., v. 46, n. 2, p. 233-242, 2003.

VIEIRA, J. P. Juvenile Mullets (Pisces, Mugilidae) in the estuary of Lagoa-dos-Patos, RS, Brazil. Copeia, v. 2, p. 409-418, 1991

VIEIRA, J. P.; MUSICK, J. A. Latitudinal patterns in diversity of fishes in warm-temperate and tropical estuarine waters of the Western Atlantic. Rev. Atlântica, v. 15 , p. $15-133,1993$.

VILAR, C. C.; SPACH, H. L.; JOYEUX, J. C. Spatial and temporal changes in the fish assemblage of a subtropical estuary in Brazil: environmental effects. J. Mar. Biol. Assoc. U. K., v. 91, n. 3, p. 635-648, 2011.

VITULE, J. R. S.; UMBRIA, S. C.; ARANHA, J. M. R. Introduction of the African catfish Clarias gariepinus (BURCHELL, 1822) into Southern Brazil. Biol. Invasions, v. 8, n. 4, p. 677-681, 2006.

ZAHORCSAK, P.; SILVANO, R. A. M.; SAZIMA, I. Feeding biology of a guild of benthivorous fishes in a sandy shore on south-eastern Brazilian coast. Rev. Bras. Biol., v. 60, n. 3, p. 511-518, 2000.

(Manuscript received 22 February 2013; revised 30 November 2013; accepted 06 December 2013)

\section{ERRATA}

\section{ANALYSIS OF FISH ASSEMBLAGES IN SECTORS ALONG A SALINITY GRADIENT BASED ON SPECIES, FAMILIES AND FUNCTIONAL GROUPS}

Na pág. 252

Onde se lê:

Fig. 1. Multidimensional ordination (MDS) based on the presence/absence of families between three salinity sectors $(\mathrm{E}=$ euhaline; $\mathrm{P}=$ polyhaline; $\mathrm{M}=$ mesohaline $)$.

Leia-se:

Fig. 1. Location of the sampling sites in the euhaline (E1-E15), polyhaline (P1-P20) and mesohaline (M1-M18) sectors of the east-west axis of the Paranaguá Estuarine Complex. 